\title{
Spatial and Temporal Structure of Receptive Fields in Primate Somatosensory Area 3b: Effects of Stimulus Scanning Direction and Orientation
}

\author{
James J. DiCarlo and Kenneth O. Johnson \\ Krieger Mind/Brain Institute, Departments of Neuroscience and Biomedical Engineering, Johns Hopkins University, \\ Baltimore, Maryland 21218
}

\begin{abstract}
This is the third in a series of studies of the neural representation of tactile spatial form in somatosensory cortical area $3 b$ of the alert monkey. We previously studied the spatial structure of $>350$ fingerpad receptive fields (RFs) with random-dot patterns scanned in one direction (DiCarlo et al., 1998) and at varying velocities (DiCarlo and Johnson, 1999). Those studies showed that area $3 \mathrm{~b}$ RFs have a wide range of spatial structures that are virtually unaffected by changes in scanning velocity. In this study, 62 area $3 b$ neurons were studied with three to eight scanning directions (58 with four or more directions). The data from all three studies are described accurately by an RF model with three components: (1) a single, central excitatory region of short duration, (2) one or more inhibitory regions, also of short duration, that are adjacent to and nearly synchronous with the
\end{abstract}

This is the third in a series of studies of the spatial and temporal response properties of area $3 b$ neurons with receptive fields (RFs) on the distal fingerpads. Each study used scanned, random-dot stimuli and regression analysis to estimate RF structure. The first study (DiCarlo et al., 1998) showed that these RFs have a single, central excitatory region and one or more flanking inhibitory regions. Because the first study used a single scanning velocity and direction, it was impossible to discriminate between spatial and temporal mechanisms.

The second study (DiCarlo and Johnson, 1999) varied scanning velocity to discriminate temporal and spatial mechanisms. The idea was that delays between the stimulus and individual response components result in displacements of their apparent skin origins that are proportional to the scanning velocity and the delays. For example, if excitatory and inhibitory effects arise from the same skin location, but the inhibition is delayed in comparison with the excitation, then its RF location will appear to trail behind the excitatory location by a distance proportional to the relative delay and the scanning velocity. A surprising result of the second study, given the extensive evidence of substantial delays between excitation and inhibition in area 3b (Andersson, 1965; Innocenti and

\footnotetext{
Received May 27, 1999; revised Oct. 6, 1999; accepted Oct 20, 1999.

This study was supported by National Institutes of Health Grant NS18787 and by the W.M. Keck Foundation. We thank Dr. S. Hsiao for helpful advice and Dr. S. Hendry for help with the laminar analysis. John Lane, Steve Patterson, and David O'Shaughnessy provided invaluable technical support.

Correspondence should be addressed to Kenneth Johnson, 338 Krieger Hall, Johns Hopkins University, 3400 North Charles Street, Baltimore, MD 21218. Email: Kenneth.Johnson@jhu.edu.

Dr. DiCarlo's present address: Howard Hughes Medical Institute and Department of Neuroscience, Baylor College of Medicine, One Baylor Plaza, Room S603, Houston, TX 77057. E-mail: dicarlo@focus.neusc.bcm.tmc.edu.

Copyright (C) 1999 Society for Neuroscience $0270-6474 / 00 / 200495-16 \$ 15.00 / 0$
}

excitation, and (3) a region of inhibition that overlaps the excitation partially or totally and is temporally delayed with respect to the first two components. The mean correlation between the observed RFs and the RFs predicted by this three-component model was 0.81 . The three-component RFs also predicted orientation sensitivity and preferred orientation to a scanned bar accurately. The orientation sensitivity was determined most strongly by the intensity of the coincident RF inhibition in relation to the excitation. Both orientation sensitivity and this ratio were stronger in the supragranular and infragranular layers than in layer IV.

Key words: receptive field; reverse correlation; somatosensory; monkey; cortex; cortical layer; orientation sensitivity

Manzoni, 1972; Gardner and Costanzo, 1980a), was that excitatory and inhibitory intensity were affected strongly, but RF spatial structure was virtually unaffected by changes in scanning velocity over the range from 20 to $80 \mathrm{~mm} / \mathrm{sec}$.

The simplest explanation for this invariance in spatial structure is that the excitatory and inhibitory effects in area $3 \mathrm{~b}$ are all brief and synchronous. But this explanation fails to account for the large changes in excitatory and inhibitory intensity with changes in scanning velocity and the studies that show substantial inhibitory delay in comparison with excitation. An alternative explanation based on the cancellation of overlapping excitation and inhibition accounts for all these observations (DiCarlo and Johnson, 1999). These two explanations make different predictions about the responses to stimuli scanned in different directions. If the excitatory and inhibitory effects are all brief and synchronous, then the RF obtained from our methods will be unaffected by scanning direction. If, however, there is substantial lagged inhibition, the RF will appear to change in predictable ways as direction changes.

In this study, random-dot patterns and bars were scanned in up to eight directions over the RF of each neuron. RFs were determined from the responses to random-dot stimulation in each direction. The central excitatory region and a portion of the inhibition of each RF were unaffected by direction. The remaining inhibition was affected in the manner expected of a temporally lagged component. The data were well described by a spatialtemporal RF model containing an excitatory component, a spatially offset, temporally synchronous inhibitory component, and a delayed inhibitory component that overlaps the excitation. Orientation sensitivity, measured separately by scanned bars, was predicted accurately by this RF model. Both orientation sensitiv- 
ity and the RF components related to orientation sensitivity were more prominent in the infragranular and supragranular layers than in layer IV.

\section{MATERIALS AND METHODS}

Animals and surgery. Two rhesus monkeys (Macaca mulatta) weighing $4-5 \mathrm{~kg}$ were used in this study. Each animal was trained to perform a visual detection task during the presentation of tactile stimuli; the purpose was to maintain the animal in a constant, alert state during recording periods. After the animal was performing the task nearly perfectly, which took a few weeks, a head-holding device and recording chamber were attached to the skull. Surgical anesthesia was induced with ketamine $\mathrm{HCl}(33 \mathrm{mg} / \mathrm{kg}$, i.m.) and maintained with pentobarbital (10 $\mathrm{mg} \cdot \mathrm{kg}^{-1} \cdot \mathrm{hr}^{-1}$, i.v.). Animal housing and all surgical and experimental procedures complied with the guidelines of the Johns Hopkins Animal Care and Use Committee and the Society for Neuroscience.

Recording. Electrophysiological recordings were done with techniques described previously (DiCarlo et al., 1998). Briefly, we recorded from neurons in area $3 \mathrm{~b}$ of three hemispheres with a multielectrode microdrive (Mountcastle et al., 1991) loaded with seven quartz-coated platinum/tungsten (90/10) electrodes (diameter, $80 \mu \mathrm{m}$; tip diameter, 4 $\mu \mathrm{m}$; impedance, $1-5 \mathrm{M} \Omega$ at $1000 \mathrm{~Hz}$ ). Each electrode was coated with one of two fluorescent dyes (DiI or DiI-C5; Molecular Probes, Eugene, $\mathrm{OR}$ ), which were used later to identify the recording locations (DiCarlo et al., 1996; see below). A continuous record of stimulus location and the times of occurrences of action potentials, stimulus events, and behavioral events were stored in a computer with an accuracy of $0.1 \mathrm{msec}$ (Johnson and Phillips, 1988). All neurons in area $3 \mathrm{~b}$ that met the following criteria were studied with the stimulus procedures described later: (1) the neuron's action potentials were well isolated from the noise; (2) the neural RF was on one of the distal fingerpads (digits 2-5); and (3) the stimulus drum (described later) and the hand could be positioned so that the RF was centered on the portion of the fingerpad in contact with the stimulus.

Stimuli. The primary stimulus patterns were arrays of raised dots distributed randomly within a rectangular region $28 \mathrm{~mm}$ wide and 250 $\mathrm{mm}$ (first monkey) or $175 \mathrm{~mm}$ (second monkey) long (for details, see DiCarlo et al., 1998). Dots were randomly distributed within this rectangular region with a mean density of $10 \mathrm{dots} / \mathrm{cm}^{2}$. Each dot was $400 \mu \mathrm{m}$ high (relief from the surface) and $500 \mu \mathrm{m}$ in diameter at its top, with sides that sloped away at $60^{\circ}$ with respect to the surface of the stimulus pattern. Random-dot patterns are unbiased in the sense that all possible patterns with the specified dot density are equally likely and the probability of a repeated pattern is virtually zero.

The dot pattern was wrapped around and glued to a cylindrical drum, $320 \mathrm{~mm}$ in circumference, which was mounted on a rotating drum stimulator (see DiCarlo and Johnson, 1999, their Fig. 1). This drum stimulator, which has three controllable degrees of freedom (drum rotation, contact force, and position along the axis of rotation), was suspended from a rotational stage with one degree of freedom and moved into position with an XYZ translation stage with three degrees of freedom (Lintech Corp., Monrovia, CA). All seven degrees of freedom were controlled by servomotors interfaced to a laboratory computer. The rotation stage that controlled the scanning direction had a laser at the axis of rotation, which was used to align the axis of rotation with the center of the skin region to be stimulated. Mounted below the rotational assembly was a small, one-dimensional translation stage that moved the drum along its axis of rotation. The drum was raised and lowered by a torque motor and rotated by a direct-drive servo motor that produced no detectable vibration. The drum axis intersected the axis of the rotation stage; therefore the drum pivoted around the center of the skin contact region when the scanning direction was changed. All aspects of the motion were programmed to ensure that nearly the same stimulus surface was scanned over the neuron's RF in each scanning direction (see Fig. 1).

After one or more neurons with overlapping RF locations were isolated with one or more of the electrodes, the drum with the random-dot pattern was positioned over the fingerpad so that all the RFs were in the cutaneous region contacting the drum surface. The scanning velocity was $40 \mathrm{~mm} / \mathrm{sec}$ in all scanning directions. Contact force was set at $0.3 \mathrm{~N}$ (Johnson and Phillips, 1988).

The experimental design called for stimuli to be scanned in four or eight directions: 0 (proximal-to-distal), 45, 90 (left-to-right), 135, 180 (distal-to-proximal), 225, 270 (right-to-left), and $315^{\circ}$. Because the stim- ulus sequence was lengthy and not all neurons could be held for the entire sequence, the stimulus order $(0,180,90,270,45,135,225$, and $315^{\circ}$ ) was designed to produce the greatest possible range of directions at any stopping point. When four scanning directions were used, the order was $0,90,180$, and $270^{\circ}$. When the neural recording was still stable after all scanning directions had been studied, the $0^{\circ}$ direction was repeated.

The drum was positioned initially so the cutaneous contact region was within the random-dot pattern and the center of the contact region was $\sim 5 \mathrm{~mm}$ from the edge of the long side of the pattern. After each revolution the drum was stepped $400 \mu \mathrm{m}$ along its axis of rotation. The drum typically completed $25-40$ revolutions in each scanning direction and therefore stepped $10-16 \mathrm{~mm}$ across the pattern. Before each new scanning direction, the drum was translated back to its starting location to ensure that approximately the same random-dot pattern was scanned across the neuron's RF in each scanning direction. Four directions and a repeated first direction required $20 \mathrm{~min}$; eight directions required $36 \mathrm{~min}$.

Before applying the stimulator, a thin latex sheet $(\sim 50 \mu \mathrm{m}$ thick; Carter-Wallace, New York, NY) was positioned over the fingerpad, and glycerin was applied to the drum's surface to eliminate friction between the drum and the latex. The latex sheet was tethered in all directions by gluing its edges to a 20-mm-diameter aperture in the center of a thin (6 $\mu \mathrm{m}), 10 \times 10 \mathrm{~cm}$ Mylar sheet (DuPont, Wilmington, DE). A square Plexiglas frame positioned horizontally over the fingerpad (see DiCarlo and Johnson, 1999, their Fig. 1) supported the Mylar sheet. The frame was lowered with a micrometer until the latex sheet contacted the fingerpad with a normal force of $0.1 \mathrm{~N}$. The purpose of the Mylar sheet, which was essentially inextensible, was to prevent horizontal skin displacement when the scanning direction changed. Horizontal skin displacement produced by changes in scanning direction was $<1 \mathrm{~mm}$. The thin latex sheet between the stimulus and the skin surface (identical to the latex sheet used by DiCarlo and Johnson, 1999) allowed the stimulus features to be transmitted to the skin. Control studies showed that the firing rates, response structures, and RFs of most area $3 \mathrm{~b}$ neurons were unaffected by the latex intermediate (J. J. DiCarlo and K. O. Johnson, unpublished observations). RFs estimated in the same scanning direction with and without the latex intermediate are shown in Figures 3-7.

Responses. The action potential times were recorded with a resolution of $0.1 \mathrm{msec}$. The data collected at each scanning direction from each neuron were maintained as a separate data set. Within each of these data sets the action potentials were assigned two-dimensional $(x, y)$ locations in relation to the drum surface (Johnson and Phillips, 1988). The $x$ location (distance in the scanning direction from the beginning of the random-dot pattern) was obtained from a digital shaft encoder. The $y$ location was determined by the axial (horizontal) drum position. The method's precision is better than $8 \mu \mathrm{m}$ in the scanning direction and 2.5 $\mu \mathrm{m}$ in the axial direction (Johnson and Phillips, 1988). Two-dimensional raster plots of individual data sets are shown in Figure 1.

Receptive field estimation. The responses in each scanning direction were used to obtain independent linear RF estimates for each direction. The method used to estimate each RF was the same as in both our previous studies (DiCarlo et al., 1998; DiCarlo and Johnson, 1999) and is therefore only described briefly.

The firing pattern evoked by the random-dot stimulus at each scanning direction was used to infer the two-dimensional pattern of excitation and inhibition on the skin surface. We assumed that each small region of skin had a positive, negative, or zero effect on the firing rate when stimulated and that the instantaneous firing rate was equal to the sum of these effects. Specifically, we subdivided a $10 \times 10 \mathrm{~mm}$ square region of skin containing the RF into a grid of $625(25 \times 25)$ subregions, each $0.4 \times 0.4$ $\mathrm{mm}$ square. We then determined the contribution of each subregion to the observed neural response with multiple regression. The grid of 625 positive (excitatory) and negative (inhibitory) values are the weights that produce the best (least-squared error) approximation of the observed firing rates when convolved with the stimulus pattern. The units of these weights are impulses per second (ips) per millimeter of indentation. The integral of the excitatory (inhibitory) weights is referred to as the excitatory (inhibitory) mass of the RF (see DiCarlo et al., 1998). The relationship of this RF estimation method to other methods that have been used is discussed by DiCarlo and Johnson (1999).

The three-component $R F$ model. To describe the effect of scanning direction on each neuron's RF, we constructed an RF model with three Gaussian subfields, one for each of the three RF components described 
in the introductory remarks. We refer to these three components as the (1) excitatory, (2) fixed inhibitory, and (3) lagged inhibitory components. Each Gaussian subfield has the form:

$$
\begin{aligned}
& G(x, y)=a \cdot e^{-\frac{1}{2}\left(L^{T} S^{-1} L\right)} \\
& L=\left[\begin{array}{c}
x-\mu_{\mathrm{x}}-\mathbf{v}_{\mathrm{x}} \cdot \tau \\
y-\mu_{\mathrm{y}}-\mathbf{v}_{\mathrm{y}} \cdot \tau
\end{array}\right] S=\left[\begin{array}{cc}
\sigma_{\mathrm{x}}^{2} & \rho \sigma_{\mathrm{x}} \sigma_{\mathrm{y}} \\
\rho \sigma_{\mathrm{x}} \sigma_{\mathrm{y}} & \sigma_{\mathrm{y}}^{2}
\end{array}\right],
\end{aligned}
$$

in which $(x, y)$ represent mediolateral and proximodistal locations on the skin surface, $\left(\mu_{\mathrm{x}}, \mu_{\mathrm{y}}\right)$ represents the center of the subfield, $\left(\mathbf{v}_{\mathrm{x}}, \mathbf{v}_{\mathrm{y}}\right)$ represents the stimulus velocity vector, and $\tau$ represents the delay of the peak of excitation or inhibition with respect to skin stimulation. The parameters $a, \sigma_{\mathrm{x}}, \sigma_{\mathrm{y}}$, and $\rho$ together specify the amplitude, spread, orientation, and elongation of the excitatory $(a>0)$ or inhibitory $(a<0)$ component represented by the Gaussian function.

Each component is delayed with respect to skin stimulation, and therefore the effect of each component is displaced from its true center by an amount and direction that is proportional to $\left(\mathbf{v}_{\mathrm{x}}, \mathbf{v}_{\mathrm{y}}\right)$ and $\tau$ (for a complete discussion, see DiCarlo and Johnson, 1999, Appendix A). In the previous study (DiCarlo and Johnson, 1999) we showed that the excitatory component is delayed by $15-20 \mathrm{msec}$ with respect to skin stimulation. In the present study we are concerned with the delay of the lagged inhibitory component with respect to the excitatory component, not its absolute value. Consequently, we position the RF obtained at each scanning direction so its excitatory center is in the center of the estimation grid (DiCarlo et al., 1998), and we set the lags for the excitatory and fixed inhibitory components to zero. The lagged inhibitory delay, $\tau$, that we report is delay with respect to the excitatory component.

In each scanning direction, the RF predicted by the three-component model is the sum of the excitatory, the fixed inhibitory, and the lagged inhibitory components (E, excitatory; IF, fixed inhibitory; and IL, lagged inhibitory):

$$
\mathrm{RF}(x, y)=G_{\mathrm{E}}(x, y)+G_{\mathrm{IF}}(x, y)+G_{\mathrm{IL}}(x, y) .
$$

Therefore the three-component model of each neuron's RF is described by 19 parameters (six parameters for each of the three Gaussian components, $\mu_{\mathrm{x}}, \mu_{\mathrm{y}}, a, \sigma_{\mathrm{x}}, \sigma_{\mathrm{y}}$, and $\rho$, plus the lag, $\tau$, for the lagged inhibitory component). A single solution was made to fit the RFs for all scanning directions (and velocities; see below). Because this model is nonlinear in the parameters, an iterative, gradient descent method was used to determine the best (least-squared error) parameters (Press et al., 1992). To account for slight RF misalignment between scanning directions, we also allowed two alignment parameters (one for the $x$ direction and one for the $y$ direction) for the RF obtained in each scanning direction (except the first RF). In practice, the alignment adjustments were $<1 \mathrm{~mm}$ in each direction. When there was more than one region of fixed inhibition, the iterative procedure selected the region with greater intensity.

The data from the present study include the effects of changes in direction. Our previous study (DiCarlo and Johnson, 1999) provides a detailed characterization of the effects of velocity. To make the model fit everything that we know about these RFs, we required that the solutions behave in the same way as do area 3 b RFs when the velocity changes. In particular, a change in scanning velocity produces virtually no change in the spatial structure of area $3 \mathrm{~b}$ RFs, but it does affect the excitatory and inhibitory intensities. The observed pixel-by-pixel correlations between RFs determined at 20 and 40, 40 and 80 , and 20 and $80 \mathrm{~mm} / \mathrm{sec}$ averaged $0.85,0.80$, and 0.76 , respectively. These were nearly identical to the average correlations expected for repeated observations of the same RF if there was no change in its spatial structure $(0.87,0.81$, and 0.82 , respectively; for details, see DiCarlo and Johnson, 1999). Doubling scanning velocity produced a $61 \%$ increase in inhibitory mass on average; the comparable figure for excitatory mass was $41 \%$. Excitatory (inhibitory) mass was measured as the integral of the absolute value of excitatory (inhibitory) RF values over the excitatory (inhibitory) area of the RF. The SEM in both cases was $6 \%$. We forced the model to account for these velocity effects by creating two additional RFs in each direction with the same RF spatial structure as at $40 \mathrm{~mm} / \mathrm{sec}$ but with the excitatory and inhibitory intensities scaled appropriately for 20 and $80 \mathrm{~mm} / \mathrm{sec}$. Thus the model with 19 parameters was required to fit three times as many RFs as scanning directions (e.g., 24 RFs if eight scanning directions were used).
The solution was unstable when scanning direction had a small effect on RF structure in comparison with the variability of the RF estimates. That occurs, for example, when the lag $(\tau)$ is small or the lagged inhibitory mass is small. In those cases the iterative procedure sets the model lag to a very small value or zero, but when that is so the lagged inhibition ( $G_{\mathrm{IL}}$ in Eq. 2) is almost fixed and is functionally indistinguishable from the fixed inhibition $\left(G_{\mathrm{IF}}\right)$. As a result the observed inhibition can be accounted for by either component alone or a combination of the two; a wide range of solutions for $G_{\mathrm{IL}}$ and $G_{\mathrm{IF}}$ produce the same RF. The model was used to estimate the relative intensities of the lagged and fixed inhibitory components only when variation in the $G_{\mathrm{IF}}$ mass (over all initial parameter values) was $<20 \%$ of the mean $G_{\mathrm{IL}}$ mass.

Orientation sensitivity. Some neurons were studied with three raised bars as well as with the random-dot patterns. The bars were constructed from photosensitive plastic sheets, mounted on a 320-mm-circumference drum, and applied to the skin in exactly the same way as the random dots. Each bar was $400 \mu \mathrm{m}$ high (relief from the surface) and $500 \mu \mathrm{m}$ wide at its top, with sides that sloped away at $60^{\circ}$ with respect to the surface of the stimulus pattern. The bars were oriented orthogonal to and at $\pm 45^{\circ}$ (clockwise) in relation to the scanning direction. The bars were long enough (at least $30 \mathrm{~mm}$ long) so that the end of a bar never touched the skin and were spaced far enough apart (at least $30 \mathrm{~mm}$ between bars) so that two bars were never on the skin at the same time. The bar pattern was scanned over the neuron's RF 8-25 times in each of eight scan directions separated by $45^{\circ}$ as in the random-dot scans.

The reason for including bars at $\pm 45^{\circ}$ was to examine the responses for any interaction between scanning direction and stimulus orientation. In fact, the orientation sensitivities and preferred orientations obtained from the three bars were essentially identical. For example, if the peak response to the orthogonal bar occurred with a proximal-to-distal scan, then the peak response to the bar oriented at $-45^{\circ}$ occurred when the scanning direction was rotated $+45^{\circ}$ from the proximal-to-distal scan. The data from all three bars were combined for this reason.

A summary measure of the response to each bar scanned in each direction ( 24 response values) was computed by binning the neuron's spikes from all repeated scans ( $8-25$ repetitions) in $400 \mu \mathrm{m}$ (10 msec) bins, smoothing the resulting histogram (70 msec boxcar filter), and taking the response to be the peak of the resulting histogram (e.g., see Fig. 12). The orientation sensitivity was measured by plotting the data in polar coordinates and fitting an ellipse to the data points (i.e., the ellipse with least mean squared error measured on the radial dimension). In all those cases in which a neuron was studied with both the bars and the random-dot pattern, the responses to the bars were predicted by convolving the three-component RF model with a bar $(400 \mu \mathrm{m}$ in relief and 400 $\mu \mathrm{m}$ in width) moving orthogonal to its long axis. Sixteen evenly spaced scanning directions were simulated, and the response in each direction was measured as the peak value of the simulated response histogram smoothed with a $70 \mathrm{msec}$ wide boxcar filter (i.e., exactly the same measure used to describe the actual neural data). An ellipse was fitted to the predicted data in exactly the same way as the observed data (see Fig. 12).

Histology. Histological methods and the methods for coating the electrodes with fluorescent dyes (DiI or DiI-C5) are described by DiCarlo et al. (1996). Tissue sections were $50 \mu \mathrm{m}$ thick and were oriented approximately parallel to the seven electrode tracks made each day and orthogonal to the central sulcus. The fluorescent dye track left by each electrode typically traversed several (e.g., 5-10) serial tissue sections as it descended into area $3 \mathrm{~b}$. The entire track of each electrode penetration was captured in a three-dimensional computer data format by tracing the portion of the dye track visible on each serial tissue section and then aligning the tissue sections to a set of four common reference points (Neurolucida). DiCarlo et al. (1996) reported that DiI and DiI-C5 stained the tracks to the point of deepest penetration in 16 of 16 tracks under circumstances like those in this experiment (total driving time to deepest point, $<3 \mathrm{hr}$ ). Therefore, the deepest point at which dye could be detected was also marked in the data file, and it was linked to the deepest microdrive reading. Because the electrode penetrations traveled nearly parallel to the cortical layers (see DiCarlo et al., 1996, their Fig. 1), small errors in the accuracy of the depth measurements are unlikely to produce errors in laminar assignment. Single-unit recordings were assigned a location within one of the serial sections based on the distance from the recording site to the point of deepest penetration. The recording location was assigned to a cortical area and layer using the criteria of Powell and Mountcastle (1959). 


\section{RESULTS}

We studied all well isolated neurons in area $3 \mathrm{~b}$ that had an RF on one of the distal fingerpads. A neuron was excluded only if the finger and the stimulator could not be positioned to bring the RF, mapped with a manual probe, well within the contact region between the skin and stimulus surface. Even neurons that were marginally responsive to manual probing were studied with the idea that the random-dot pattern might uncover responsiveness that was not evident with simpler stimuli. One hundred sixty-two neurons in three hemispheres of two monkeys were studied with random-dot patterns scanned in at least two directions.

The essential result was that all area $3 b$ RFs were affected by the scanning direction. After examination of the entire population of neurons, it became clear that the most parsimonious hypothesis that might explain these effects was that each neuron's RFs had three components: (1) a region of excitation, (2) one or more regions of inhibition whose locations were fixed in relation to the excitatory center and were unaffected by changes in scanning direction, and (3) a region of inhibition whose position depended on the scanning direction. In the following sections, we describe the neural response and RF data that led to this threecomponent hypothesis. We then generate a quantitative model consisting of three Gaussian subfields to test the adequacy of this hypothesis.

All area $3 \mathrm{~b}$ neuronal responses were affected by the scanning direction. This is the expected result unless a neuron's RF is circularly symmetric; almost none of the RFs that we have studied in area 3b are circularly symmetric (DiCarlo et al., 1998). A typical neuronal response is illustrated in Figure 1. The designations left and right in all figures in this paper refer to the skin of the fingerpad as if viewed through the back of the finger with the fingertips pointing vertically (see Fig. 1 legend). When the stimulus pattern was scanned from proximal to distal $(\mathrm{P} \rightarrow \mathrm{D})$ over the $\mathrm{RF}$, the neuron responded best when dots clustered in groups with distal-right to proximal-left orientations in relation to the fingerpad passed over the neuron's RF. That response pattern suggests that the RF has regions of excitation and inhibition offset from each other in the orthogonal direction (distal-left to proximal-right); that is, in fact, what emerged when the RF was estimated (Fig. 1, RF to the right of the top raster). If the RF structure were unaffected by scanning direction, the response patterns in different scanning directions would appear the same but simply rotated to match the change in alignment between the finger and the stimulus pattern (i.e., the neuron would always respond best to dot clusters with a distal-right to proximal-left orientation on the skin). When the pattern was rotated $90^{\circ}$ and scanned from left to right (Fig. 1, second raster), the response was as expected. The $\mathrm{D} \rightarrow \mathrm{P}$ and $\mathrm{R} \rightarrow \mathrm{L}$ scans did not, however, produce the response expected from an RF with fixed structure. During the $\mathrm{D} \rightarrow \mathrm{P}$ scan (Fig. 1, third raster), the neuron responded best to clusters with a "distal-right to proximal-left" orientation, as before and also to some clusters with the orthogonal orientation (distal-left to proximal-right). During the $\mathrm{R} \rightarrow \mathrm{L}$ scan (Fig. 1 , bottom raster) the neuron responded best to dot clusters with the latter orientation (distal-left to proximal-right). These are not the responses expected from a neuron whose excitatory and inhibitory RF structure is fixed in relation to the skin. Some aspects of the response were invariant with scanning direction, and some were not.

The reason for the change in response properties between scanning directions can be seen by examining the RFs in Figure 1.
The RFs change with scanning direction. For example, RFs derived from two scanning directions $(\mathrm{L} \rightarrow \mathrm{R}$ and $\mathrm{D} \rightarrow \mathrm{P})$ have two regions of inhibition flanking the central region of excitation, whereas the RFs derived from the other two scanning directions $(\mathrm{P} \rightarrow \mathrm{D}$ and $\mathrm{R} \rightarrow \mathrm{L})$ have only a single region of inhibition. Close inspection of the four RFs in Figure 1 shows that each contains a central excitatory region and an inhibitory region distal to and left of the excitatory region. Each RF also contains an inhibitory region that lags behind the excitatory region in the scanning direction. Because the leftmost RF plots in Figure 1 are displayed in the same orientation as the stimulus patterns, this lagged inhibitory component appears in the left side of each of these RF plots. The three RF components evident in Figure 1, were evident in most of the neurons that we studied. We refer to these three components as the (1) excitatory, (2) fixed inhibitory, and (3) lagged inhibitory components.

To test the adequacy of this three-component description on the entire population, the RFs of each neuron in the study were fitted with the model illustrated in Figure 2 in which the three RF components are represented by Gaussian functions (see Materials and Methods). The aim was not to capture the exact shape of the individual components but instead to assess the validity of the three-component description and to obtain an estimate of the locations, sizes, and magnitudes of the three components. In fitting the model, the Gaussian functions representing the RF components were allowed to vary in intensity, spatial location, and spatial spread (i.e., overall area including possible spatial elongation and orientation). The two inhibitory components were allowed to overlap the excitation, just as inhibition and excitation are known to overlap in the RFs of area $3 b$ neurons (Laskin and Spencer, 1979; Gardner and Costanzo, 1980a). The center of the lagged inhibition trailed (in the scanning direction) behind a center at a fixed skin location, which we will refer to as the lag center (see Fig. 2). The lag center was not forced to coincide with the center of excitation. The trailing distance was proportional to the temporal lag and the scanning velocity. Both the lag center and the temporal lag itself were model parameters that were adjusted to fit the data. If the RF had been mapped with a dynamic stimulus that had no overall group motion, the center of the lagged inhibition would lie at the lag center. A physiological realization of the lagged inhibition is an inhibitory region centered at the lag center whose inhibitory effect is delayed with respect to the excitatory effect.

An iterative gradient descent method (Press et al., 1992) was used to find the three Gaussian functions, the lag center, and the temporal lag that provided the best, least-squared description of all the RFs obtained from each neuron (see Materials and Methods). Although the number of free parameters in the model is moderately large (19), the number of data being fitted is much larger; on average each model fitted $3200 \mathrm{RF}$ values (625 RF values in 5.1 RFs on average). To ensure a reliable solution we required that at least two RFs obtained in scanning directions separated by $\geq 90^{\circ}$ had RF noise indices $<30 \%$ (DiCarlo et al., 1998). Data from 78 neurons met this criterion, and all but two provided reliable (noise index, $<30 \%$ ) RF estimates in at least three directions. RFs obtained in other scanning directions were included when their noise indices were $<50 \%$. The average number of RF estimates used to determine the model parameters was 5.1 (median, 4). We also required that the solution be stable over a wide range of initial parameter values (see Materials and Methods). This eliminated 16 neurons, leaving 62 for the bulk of the analyses presented here. 


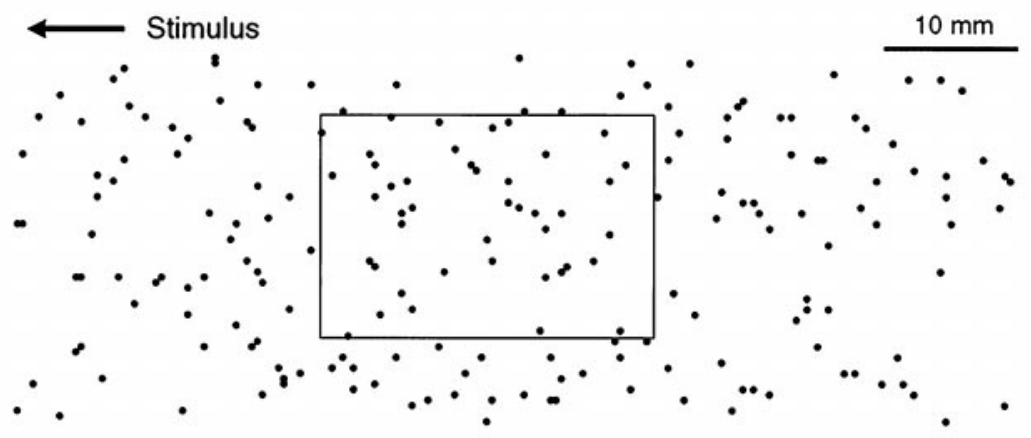

Response to stimulus scanned proximal to distal

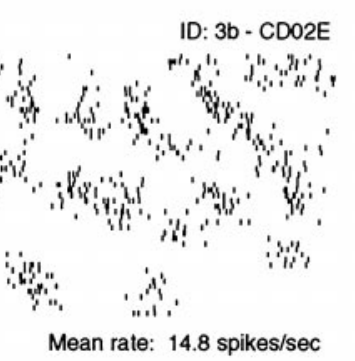

Receptive field
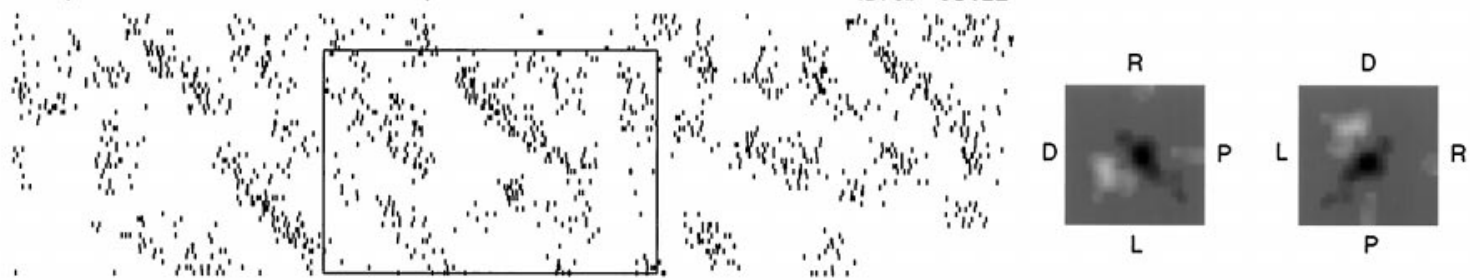

Left to right
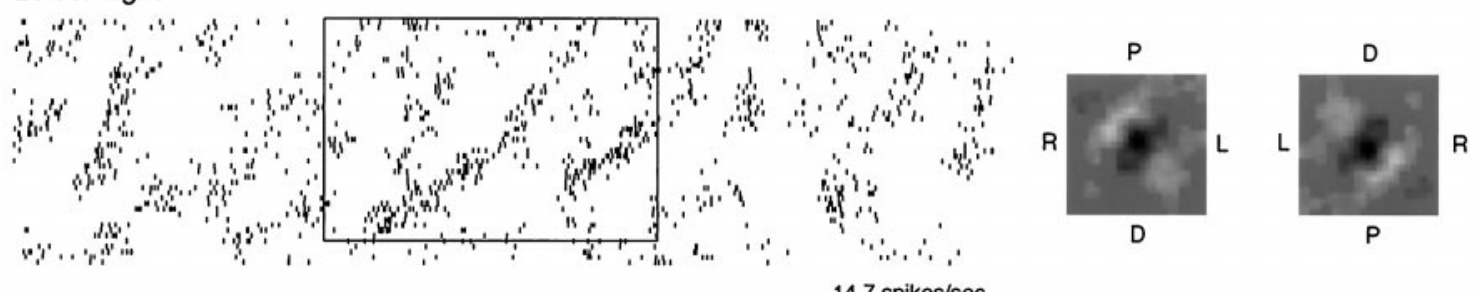

\section{Distal to proximal}

14.7 spikes/sec
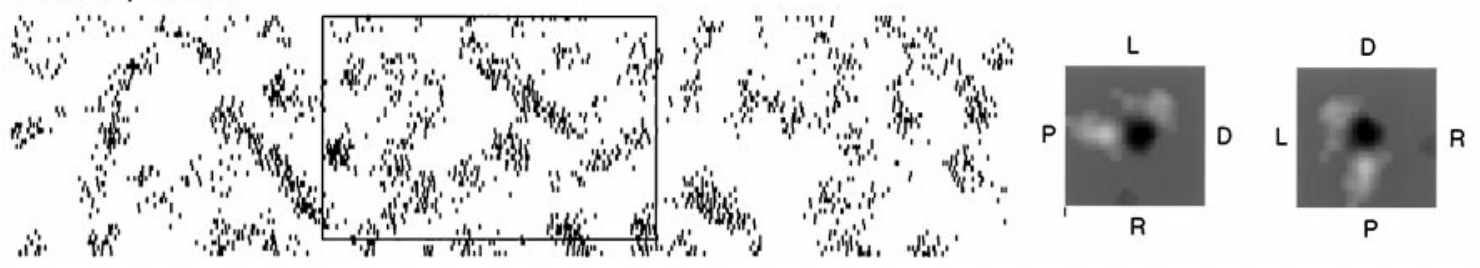

Right to left
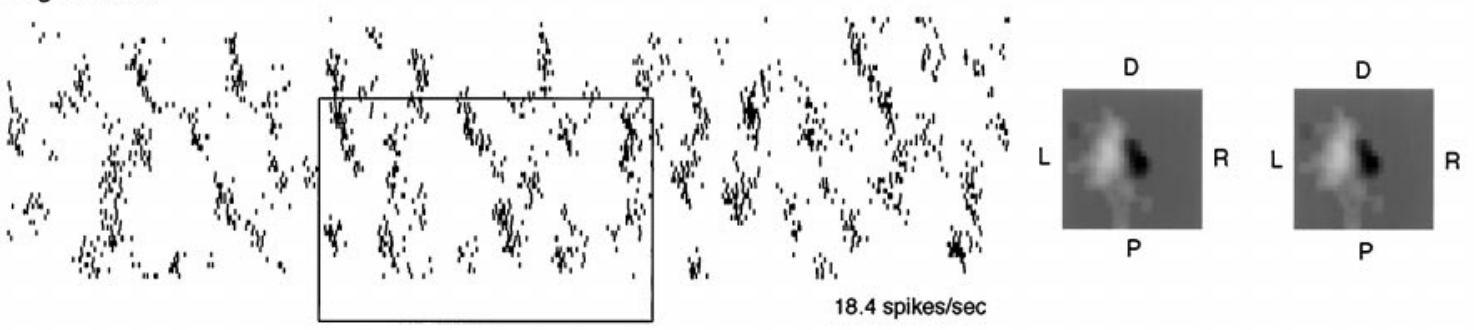

Figure 1. Effect of scanning direction on the response and RF of a typical area $3 \mathrm{~b}$ neuron. Part of the random-dot pattern ( $\sim 40 \%$ of the entire pattern) is shown at the top. Each dot on the plot illustrates the location of a stimulus element, $400 \mu \mathrm{m}$ in relief and $500 \mu \mathrm{m}$ in diameter. The neuron's responses in each scanning direction is shown as a spatial raster. Each tick mark represents a single action potential plotted at the stimulus position (top) when the action potential occurred (time flows from left to right across each raster). The box in each raster shows the neuron's response in each scanning direction to the same stimulus region (also identified with a box). The RF estimated from the response to each scanning direction is shown in two orientations on the right side of each raster. Each RF is plotted as if viewing the skin through the back of the finger (i.e., from the neuron's point of view). RFs in the left column are plotted so the finger orientation in relation to the random-dot pattern (top panel) is the same as in the experiment; RFs in the right column are plotted so the finger points toward the top of the figure (see labels next to each RF; $L$ indicates the left side of the finger when viewed through the dorsum of the finger with the tip pointing up). Each RF is represented by a $10 \times 10 \mathrm{~mm}$ gray scale image in which bins that are darker than the gray background represent skin regions in which raised stimuli (dots) had an excitatory effect on the neuron's response; bins that are lighter than the gray background represent skin regions in which raised stimuli had an inhibitory effect on the neuron's response. The peak excitatory values in the four RFs are (top to bottom) 50,39, 77, and $83 \mathrm{ips} / \mathrm{mm}$. The corresponding peak inhibitory values are 31, 28, 53, and $67 \mathrm{ips} / \mathrm{mm}$. Pattern motion in relation to the skin can be visualized by placing a fingerpad on the random-dot pattern in the orientation specified by the labels in the leftmost RF and scanning the finger from left to right across the pattern. For example, for the proximal-to-distal scanning direction (top spike raster), the finger should be placed on the stimulus pattern (top panel), pointed toward the left side of the page, and then scanned to the right side of the page. 


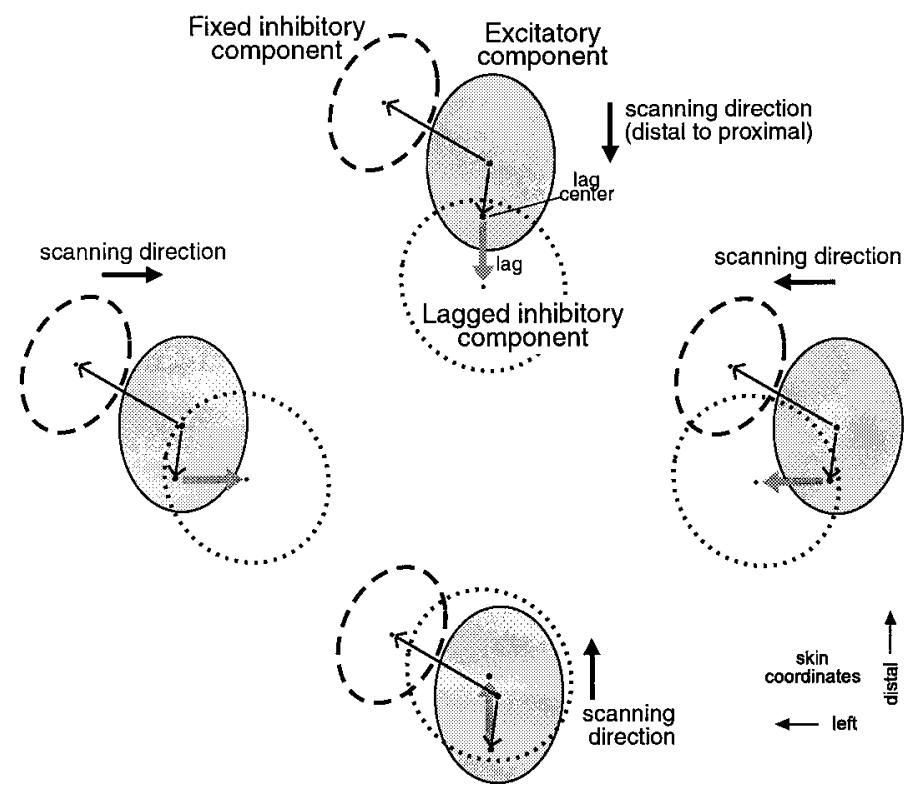

Figure 2. Three-component Gaussian model. Three ellipses in each panel represent isoamplitude contours around Gaussian functions describing three RF components (excitatory, fixed inhibitory, and lagged inhibitory). The RF predicted by the model in each scanning direction (i.e., each panel) is the sum of these three Gaussian functions. Only the lagged inhibitory component changes its apparent RF location as scanning direction changes. This change in apparent RF location is the expected change if the lagged inhibitory component was temporally delayed from the excitatory and fixed inhibitory component. The locations of the fixed inhibitory center and the lag center in relation to the excitatory subfield are identified by the two thin arrows originating from the center of the excitatory component. The displacement of the lagged inhibitory component from the lag center is indicated by the thick, gray arrow. The tail of the gray arrow is at the lag center; the arrow direction corresponds to the stimulus direction across the RF (i.e., scanning direction). The tip of the gray arrow specifies the apparent location of the lagged inhibitory center (see Materials and Methods for details.)

Figures 3-7 show five examples of the effects of scanning direction on RF structure and the degree to which the threecomponent model fits the data. Figure 3 displays results from the same neuron as in Figure 1. Three RF panels are displayed for each scanning direction. The left panel in each group (except the bottom group) is the RF estimated from the neuron's response in the indicated scanning direction. The middle panel is the RF predicted by the three-component model (i.e., the summed effect of the excitatory component and the two inhibitory components illustrated in Fig. 2). Visual comparison of the left and middle panels provides an indication of the degree to which the threecomponent model captures the essential spatial structure of the observed RF. The peak excitatory values range from 30 to 133 $\mathrm{imp} \cdot \mathrm{sec}^{-1} \cdot \mathrm{mm}^{-1}$; the peak inhibitory values range from 16 to $98 \mathrm{imp} \cdot \mathrm{sec}^{-1} \cdot \mathrm{mm}^{-1}$. The correspondence between observed and predicted RF values is presented later. An important RF property that is not easily visualized in the gray-scale representations of the RFs is the relative strengths of the excitation and inhibition; that is indicated in the legend of each of Figures 3-7 (as the absolute value of the ratio of the peak excitatory value to the peak inhibitory value, peak E/I ratio). The right panel in each group of three panels shows the shapes and locations of the three model RF components (in the same manner as in Fig. 2). The solid, dashed, and dotted lines are the 1.5 SD contours of the Gaussian functions that best fitted the central excitatory region, the fixed inhibitory region, and the lagged inhibitory region, respectively. The arrow in each panel shows the inhibitory lag magnitude and direction; its tail is at the lag center. Note that the lag is always in the direction of stimulus motion on the skin and that the magnitude is the same in all directions (because the velocity, $40 \mathrm{~mm} / \mathrm{sec}$, is the same in all directions). To the left of the row of RFs for the $\mathrm{P} \rightarrow \mathrm{D}$ scan (the bottom group in each figure) is the RF estimated from the neuron's response to $\mathrm{P} \rightarrow \mathrm{D}$ scanning without the thin latex intermediate used to limit the horizontal skin motion (see Materials and Methods). Comparison with the RF to its right shows that the overall spatial structure of the RF estimate was unaffected by the thin latex intermediate.

The lagged inhibitory region in the model in Figure 3 trails 0.99 $\mathrm{mm}$ behind a lag center $0.64 \mathrm{~mm}$ below (proximal) and slightly to the left of the excitatory center. If the lag is due to a temporal delay between excitation and the lagged inhibition, that delay is $25 \mathrm{msec}(0.99 \mathrm{~mm}$ at $40 \mathrm{~mm} / \mathrm{sec})$. Because the lag center is proximal to the excitatory center, the lagged inhibition overlaps and cancels a fraction of the excitation almost maximally when the scanning direction is $\mathrm{P} \rightarrow \mathrm{D}$ (Figs. 3, bottom panels, 1 top response raster). In the opposite scanning direction (Figs. 3, top panels, 1, third raster from top) the inhibition is almost maximally separated from the excitation, thus exposing the excitation almost maximally. This may explain the directional sensitivity of this neuron (mean firing rate of 24.9 spikes/sec in $\mathrm{D} \rightarrow \mathrm{P}$ direction and 14.8 spikes/sec in $\mathrm{P} \rightarrow \mathrm{D}$ direction; see Fig. 1).

Figures 4-7 illustrate four more RFs with a range of structural features. The points to note in assessing the adequacy of the three-component model are the degree to which the predicted inhibitory geometry (middle panel) matches the observed inhibitory geometry (left panel) and the degree to which the predicted inhibitory intensity matches the observed inhibitory intensity. The model predicts that the most intense inhibition will usually be produced when the stimulus is scanned along a line passing from the lag center to the center of fixed inhibition. In this case, the lagged and fixed inhibition will overlap and sum. According to the model, the opposite scanning direction should produce inhibition that is spread over a larger region and is less dense. In general, both predictions are satisfied. Note that the objective is not to determine whether the RF subfields are fitted by Gaussian functions but rather it is to assess the hypothesis that each RF is composed of subfields of three types. The prediction based on the three-component model highlights RF features that may deviate from this general hypothesis. In some cases there are consistent deviations from the Gaussian model, but in every case they consist of an additional region of fixed inhibition. Those cases indicate deviation from the three-component Gaussian model but at the same time conformity to the more general threecomponent hypothesis, which is the real object of the analysis.

Figure 4 illustrates an example in which the regions of fixed and lagged inhibition are elongated and the lag center is near the excitatory center. The lag in Figure 4, $1.23 \mathrm{~mm}$, is equivalent to a $30 \mathrm{msec}$ delay between excitation and the lagged component of inhibition. In two of the four scanning directions (scanning directions $\mathrm{R} \rightarrow \mathrm{L}$ and $\mathrm{D} \rightarrow \mathrm{P}$ ) the $\mathrm{RF}$ extracted from the response contains two regions of inhibition on opposite sides of the excitation. Comparison with the predicted response (middle panel) shows that the model accounts well for this inhibitory pattern. In the other two directions the RF extracted from the response has only one region of inhibition distal and to the right of the excitatory center, but it is more intense than in the $\mathrm{R} \rightarrow \mathrm{L}$ and $\mathrm{D} \rightarrow \mathrm{P}$ directions. That too is well explained by summation of the 
scanning direction $\downarrow$ (distal to proximal)

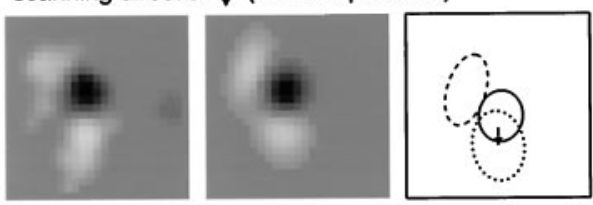

ID: 3b - CD02E
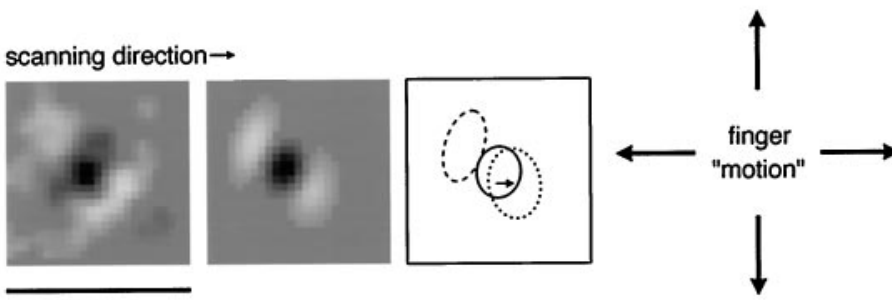

scanning direction $\leftarrow$

$10 \mathrm{~mm}$

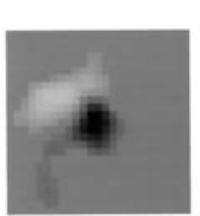

RF

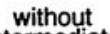

without scanning direction $\uparrow$

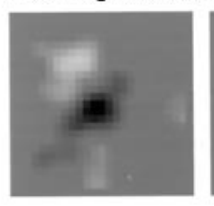

RF

with
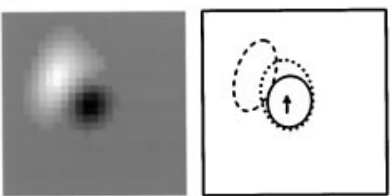

predicted RF

(three component model)

Figure 3. RFs in four scanning directions and model predictions. The neuron is the same as in Figure 1. The three squares in each group display the RF estimated from the raw data (left), the RF predicted by the three-component model (middle), and the positions of the model Gaussian components (right). The ellipses in the right square in each group are isoamplitude contours at 1.5 SD. The scanning direction is shown above each group. As in Figure 1 , each RF is plotted as if it were viewed through the dorsum of the finger (i.e., from the neuron's point of view) with the finger pointed toward the top of the figure; the effect of relative motion between the finger and the stimulus pattern on the RF can be visualized by placing a fingerpad in the center of the figure and sliding it along the arrow labeled finger "motion" toward the RF of interest. Note how the locations of the model's excitatory (solid ellipse) and fixed inhibitory (dashed ellipse) components are unaffected by scanning direction and, similarly, how the lagged inhibitory component (dotted ellipse) trails the lag center by a fixed distance in each direction (see Fig. 2). The arrow in each right square corresponds to the gray arrow in Figure 2 . The degree to which the model accounts for RF structure in each direction can be seen by comparing the left and middle panels in each group. The absolute values of the ratios of the peak excitatory values to the peak inhibitory values (peak E/I ratios) in the observed RFs are (clockwise from proximal to distal, bottom panel ) 1.6, 1.4, 1.5, and 1.2. The comparable predicted peak E/I ratios are $0.9,1.5,1.7$, and 1.3. The RF illustrated at the left of the bottom row was determined from responses without the latex intermediate (see Materials and Methods and Results).

lagged inhibitory component and the fixed inhibitory component in the distal, right part of the RF.

Figure 5 illustrates an RF with more than one region of fixed inhibition as well as a region of lagged inhibition. This RF has a strong fixed inhibitory component in the proximal, left part of each RF and a separate region of weaker fixed inhibition in the distal, right part of each RF. To reach the best solution, the three-component model-fitting algorithm assigned the fixed component to the stronger of the two fixed inhibitory componentsthe inhibition in the proximal, left portion of the RF. In three of the four scanning directions the main model error is the failure to account for the fixed inhibition distal and slightly to the right of the excitation. In the fourth, $\mathrm{P} \rightarrow \mathrm{D}$, scanning direction (Fig. 5, bottom) the effect of the missing distal, fixed component in the model can be seen even when it is obscured by the lagged inhibition; the model fails to match the intensity of the observed distal inhibition. These deviations from the predicted RFs could have been reduced by adding a second region of fixed inhibition to the model. Thus, deviations of this kind are consistent with the general three-component hypothesis. The lag illustrated in Figure 5 is equivalent to a delay of $30 \mathrm{msec}$ between the excitation and the lagged inhibition.
Figure 6 illustrates a neuron held long enough to obtain full scans in eight directions. The lagged inhibitory area in this example is large compared with the excitatory area, which produces something close to surround inhibition in some scanning directions. Because the lagged inhibition is so large, there is overlap between the fixed and lagged inhibition in several scan directions. The intense inhibition in the distal, left part of the RFs derived from the responses in three directions (Fig. 6, three RFs in the bottom right quadrant) is accounted for well by summation between the model's overlapping fixed and lagged inhibition. The lag illustrated in Figure 6 is equivalent to a $25 \mathrm{msec}$ delay between excitation and inhibition.

Figure 7 illustrates a neuron in which the excitatory area is small, both the fixed and the lagged inhibitory areas are large when compared with the excitatory area, and both regions overlap the excitatory region. The lagged inhibitory area is 3.6 times larger than the excitatory area, and its mass is 3.5 times larger than the fixed inhibitory mass. The lag center is offset from the excitatory center by a distance that is a large fraction of the radius of the excitatory area $(0.45 \times)$. The primary discrepancy between the model and the observed data in this case is the failure to account for a region of fixed inhibition in the proximal-right part 
scanning direction $\downarrow$ (distal to proximal)

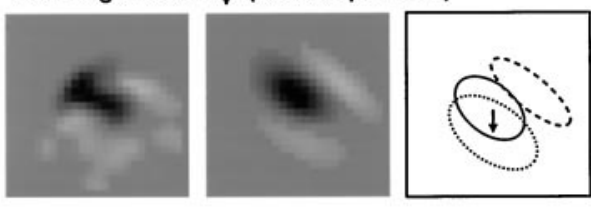

ID: $3 b-C D 02 V$
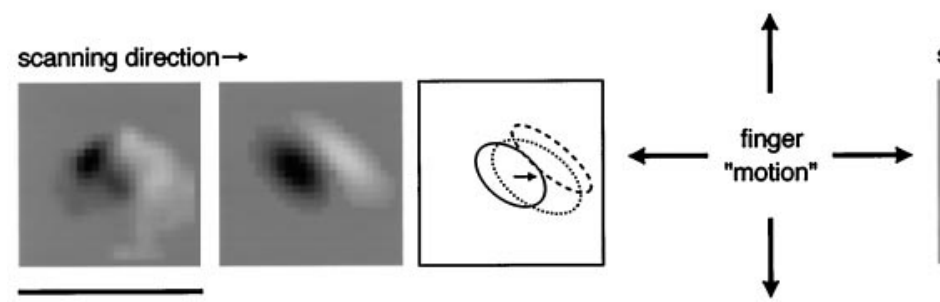

scanning direction $\leftarrow$

$10 \mathrm{~mm}$

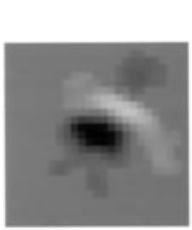

RF

without
intermediate scanning direction $\uparrow$

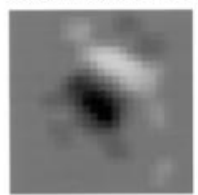

RF

with
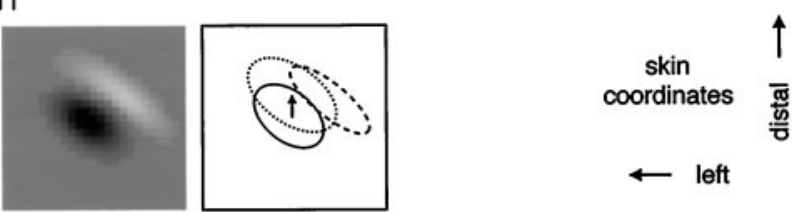

predicted RF

(three component model)

Figure 4. RF example in which the regions of fixed and lagged inhibition are elongated and the lag center is near the excitatory center. The observed (predicted) peak E/I ratios are (clockwise from proximal to distal) 1.4 (1.8), 2.1 (2.6), 2.6 (2.7), and 2.4 (2.6). Details as in Figure 3.

scanning direction $\downarrow$ (distal to proximal)
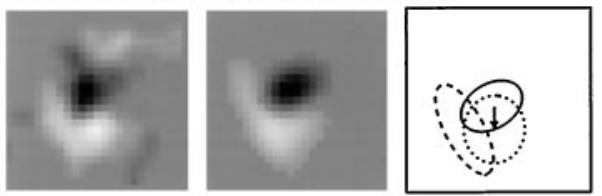

ID: 3b - CD02Y

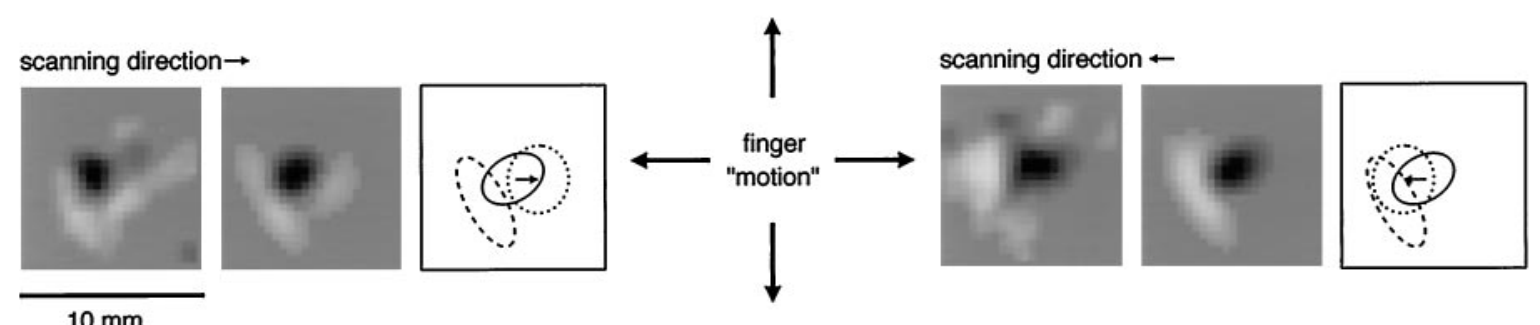

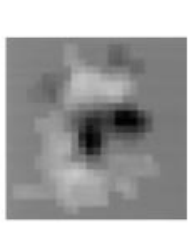

RF

without intermediate scanning direction $\uparrow$
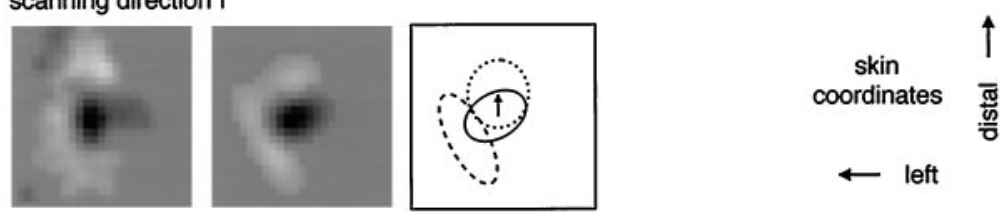

\section{predicted RF}

(three component model)

Figure 5. RF example in which there is more than one region of fixed inhibition. The observed (predicted) peak E/I ratios are (clockwise from proximal to distal) 1.7 (2.6), 2.5 (2.6), 1.2 (1.6), and 1.5 (1.8). Details as in Figure 3. 


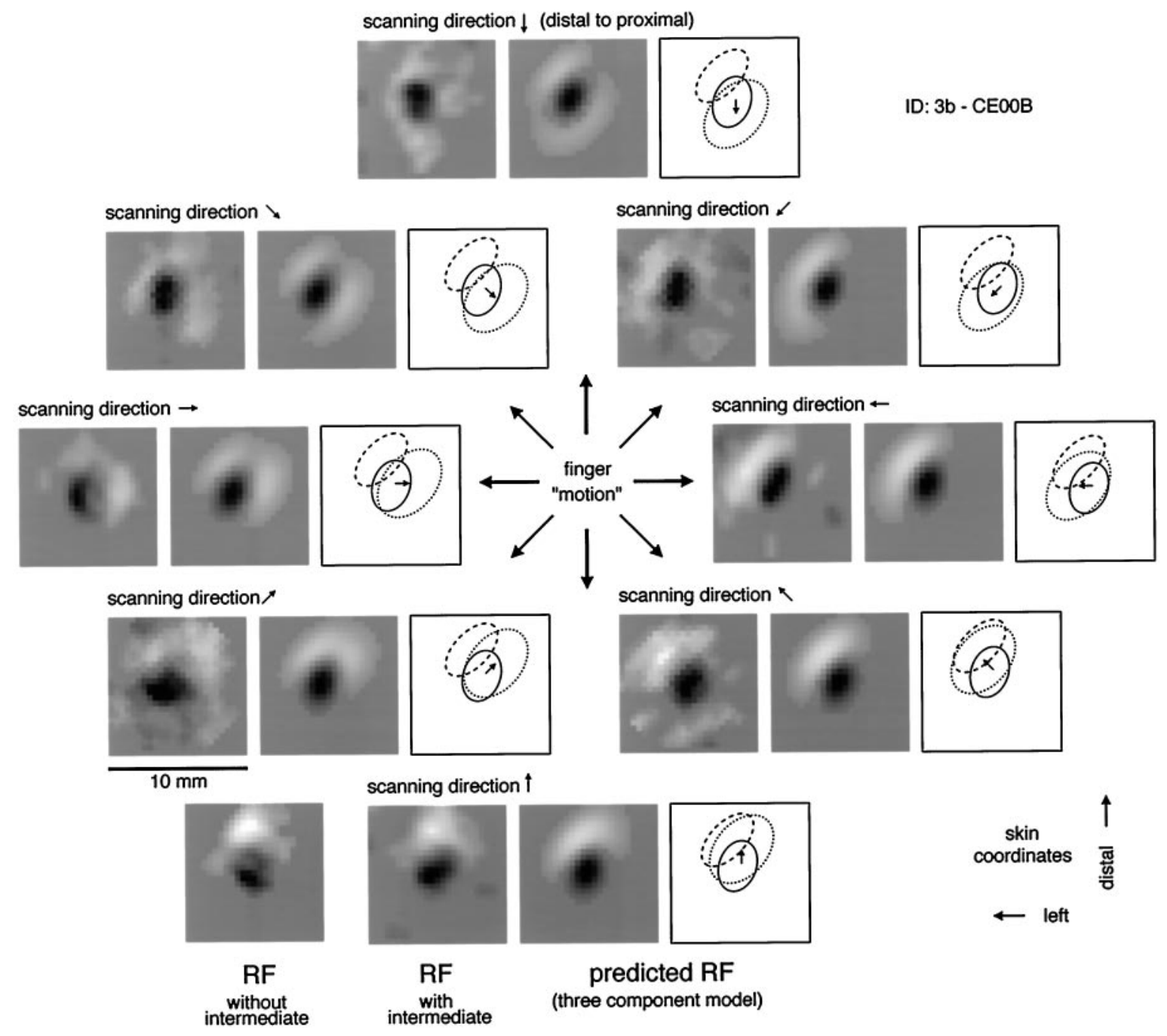

Figure 6. RF example in which a neuron was held long enough to obtain full scans in eight directions and the lagged inhibitory area is large in comparison with the excitatory area. The observed (predicted) peak E/I ratios are (clockwise from proximal to distal) 1.1 (1.2), 1.7 (1.7), 1.8 (1.9), 2.5 (2.2), 2.2 (2.2), 1.9 (1.7), 1.2 (1.3), and 1.1 (1.2). Details as in Figure 3, except that eight scanning directions were studied.

of the RF. In comparing the predicted and observed RFs, it can be seen that there is a region of inhibition in the proximal-right part of each RF that is stronger than predicted by the model. As before, that could have been rectified by allowing the model to incorporate a second region of fixed inhibition. The lag illustrated in Figure 7 is equivalent to an $18 \mathrm{msec}$ delay.

\section{Goodness of fit}

Figures 3-7 provide a qualitative summary of typical fits between the three-Gaussian model and RFs derived from responses in multiple scanning directions. Product-moment correlation coefficients displayed in Figure 8 provide a quantitative summary of the fits for all neurons studied. Specifically, each observed RF bin value was paired with the corresponding RF bin value predicted by the three-Gaussian model, and the correlation between all such pairs for a single neuron was computed. On average, 3200 RF points contributed to each correlation calculation (i.e., $625 \mathrm{RF}$ bin values in each of 5.1 scanning directions, on average). The mean correlation was 0.81 (SD, 0.09). If the model had allowed for two regions of fixed inhibition, many correlations would have been higher. The high correlation between the predicted and observed RFs is remarkable for several reasons: (1) the Gaussian components were not intended to capture the details of the shapes of the RF regions; (2) the three-Gaussian model includes only 19 parameters to describe up to $5000 \mathrm{RF}$ bin values (8 scanning directions $\times 625$ bin values in each RF); and (3) noise in the observed RFs accounts for a large part of the lack of correlation between the predicted and observed RFs (DiCarlo et al., 1998, their Fig. 12).

\section{Summary of the three response components}

No simple graphical summary of the RF components that we could find fully captured the range of RF structures. (The 19 parameters that describe the three RF components for all 62 neurons can be obtained from the authors.) Scatter plots of the areas and masses (intensities) of the three estimated RF components are shown in Figure 9. The distribution of excitatory areas is nearly identical to the distribution of excitatory areas in the larger sample reported earlier. The geometric mean excitatory area in the sample shown in Figure 9 is $13.1 \mathrm{~mm}^{2}$; the comparable area in the earlier study was $12.6 \mathrm{~mm}^{2}$ (DiCarlo et al., 1998). The two inhibitory areas and all the masses are larger than those 

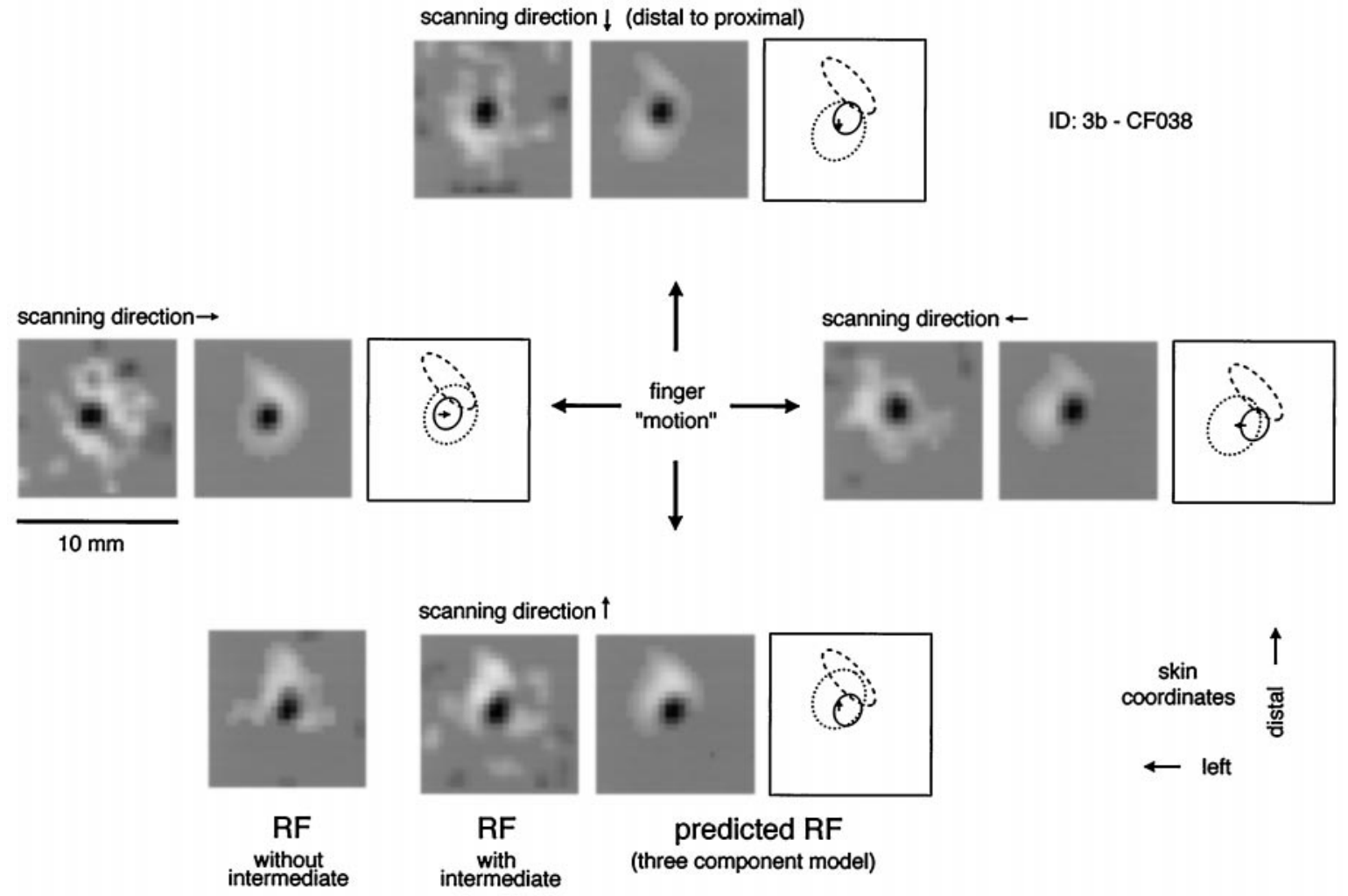

Figure 7. RF example in which the inhibitory areas are large in comparison with the excitatory area, which results in surround inhibition in some scanning directions. The observed (predicted) peak E/I ratios are (clockwise from proximal to distal) 1.3 (1.2), 1.5 (1.4), 1.6 (1.6), and 1.6 (1.6). Details as in Figure 3.

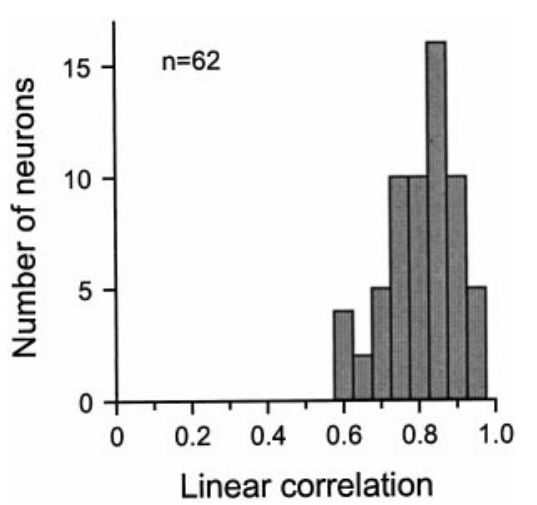

Figure 8. Model fit. The fit between the RF predicted by the threecomponent model (middle RFs in Figs. 3-7) and the observed RF (left RFs in Figs. 3-7) was computed as the correlation on a bin-by-bin basis for each of the 62 neurons. The histogram represents these 62 correlation values.

reported in the earlier study because the earlier study reported net areas and masses. When the cancellation between overlapping excitation and inhibition is accounted for, the net excitatory and inhibitory values predicted by the three-Gaussian model are similar to the observed excitatory and inhibitory values. The mean net inhibitory area predicted by the model was $16.4 \mathrm{~mm}^{2}$; the mean inhibitory area reported in the earlier study was $15.5 \mathrm{~mm}^{2}$ (DiCarlo et al., 1998). The net excitatory and inhibitory RF masses predicted by the three-Gaussian model (Figs. 3-7, middle $R F$ panels in each scanning direction) corresponded well to the observed RF masses (correlation coefficients over all RFs $=0.89$ and 0.66 for excitatory and inhibitory masses, respectively).

On average, the Gaussian fixed inhibitory area (mean, 13.4 $\mathrm{mm}^{2}$ ) was approximately equal to the excitatory area (mean, 13.1 $\mathrm{mm}^{2}$; Fig. 9, top left), but its mass (mean, 571 mass units) was only $\sim 25 \%$ as large as the average excitatory mass (mean, 2440 mass units; Fig. 9, bottom left). The average lagged inhibitory area (mean, $24.0 \mathrm{~mm}^{2}$ ) was $80 \%$ greater than the average excitatory area (Fig. 9, top middle); the average lagged inhibitory mass (1781 mass units) was $\sim 30 \%$ less than the average excitatory mass (Fig. 9, bottom middle). The average lagged-inhibitory area and mass were 80 and $200 \%$ greater than the average fixed inhibitory area and mass, respectively (Fig. 9, top and bottom right). In making these comparisons it must be borne in mind that the lagged inhibition overlapped the excitation more than did the fixed inhibition (compare Figs. 3, 8); therefore, it was canceled more by the excitation, and a smaller fraction of the lagged inhibition contributed to the net (i.e., observed) inhibition.

RF component locations have a predictable effect on a neuron's response properties. For example, the vector arising at the center of fixed inhibition and pointing toward the center of excitation defines a predicted orientation sensitivity to spatial stimulus gradients (see below). Figure 10, left panel, shows that the Gaussian fixed inhibitory component is most frequently $1-3 \mathrm{~mm}$ from the center of excitation, and it occurs on all sides of the central excitatory region. Analysis of the locations of the fixed inhibitory regions revealed a small but statistically significant lack of uniformity ( $p<0.005$; $n=62$, Kuiper's test for uniformity of a circular distribution; Mardia, 1972) consisting of a distal bias. This distal bias is not due to an asymmetry in the scanning directions used to 

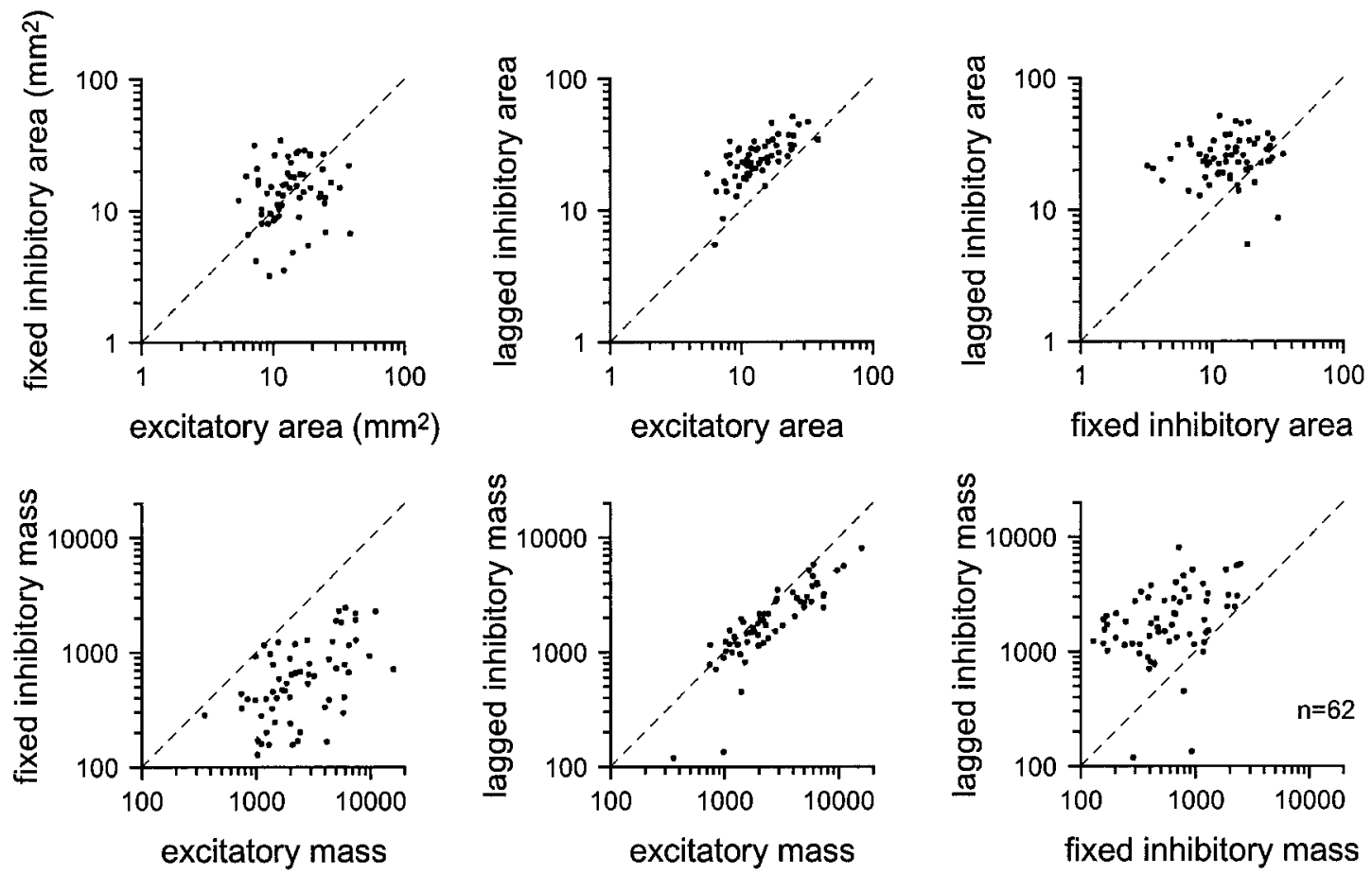

Figure 9. Areas (top row) and masses (bottom row) of the three RF response components computed from the three-component model. The area of each excitatory or inhibitory component was defined as the area within 2.15 SDs of its center. The mass of each component was defined as the sum of the absolute bin values within 2.15 SDs of its center.

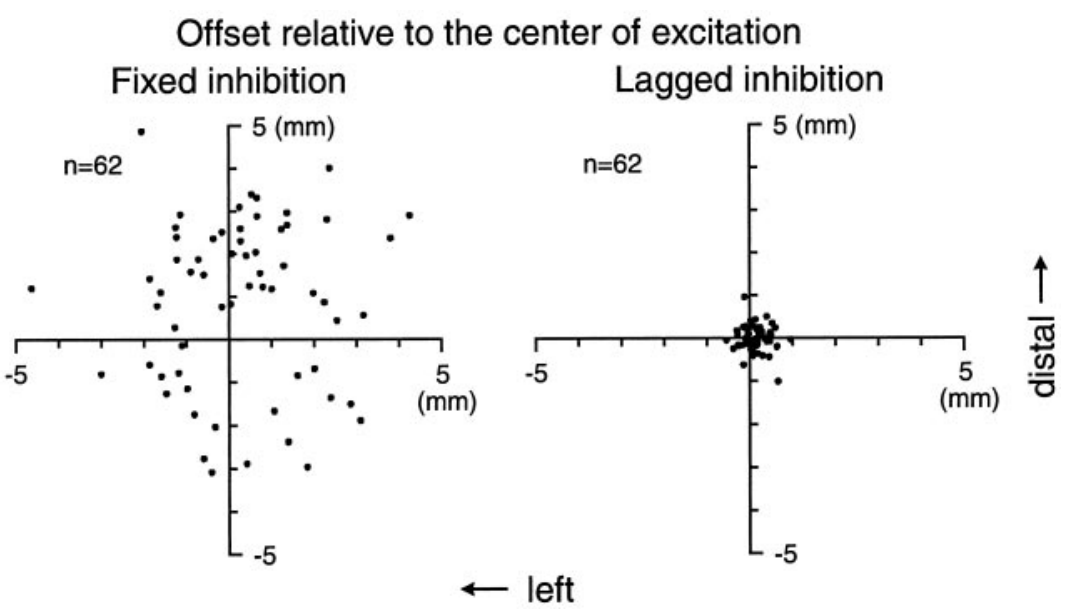

Figure 10. Inhibitory offsets from the center of excitation. The left graph displays the locations of the centers of the fixed inhibitory components in relation to the centers of the excitatory components for the entire sample of 62 neurons. The right graph displays the locations of the lag centers of the lagged inhibitory components in relation to the centers of the excitatory components. The data in both plots are displayed with the abscissa aligned from left to right. No obvious lateral bias is apparent when the data are plotted in these coordinates or in radial-ulnar coordinates. This is supported by statistical analyses (see Results).

fit the component parameters, because the nonuniformity remains statistically significant when only neurons with RFs determined in four or eight (orthogonal) directions are included ( $p<$ $0.005 ; n=41)$. Statistical tests of lateral bias showed that there was no left-right or ulnar-radial bias ( $p>0.05, t$ tests).

The lagged inhibitory component appears in the RF in each scanning direction at a position that lags a point (lag center) near the excitatory center by a fixed distance as described previously (see Fig. 2). An offset between the lag center and the center of excitation can result in directional sensitivity (Barlow and Levick, 1965); the scanning direction yielding the maximal mean firing rate should be (approximately) the direction of this offset (see Discussion). If this is true, the scanning directions producing maximal mean response rates (i.e., the preferred scanning directions) should be distributed in all directions because the lag centers are distributed in all directions around the excitatory centers $(p>0.1 ; n=62$, Kuiper's test for uniformity of a circular distribution; Mardia, 1972). However, because the lag center offsets are generally small (in comparison with the spread of the excitatory and lagged inhibitory components), the directional sensitivities should be mild for most neurons.

\section{Effect of scanning direction on firing rate}

With a few exceptions (e.g., Fig. 1), scanning direction had no discernible effect or only a small effect on firing rate. Figure 11 shows the average firing rates of 26 neurons that (1) were studied long enough to obtain scans in all eight directions plus a repeated scan in the original proximal-to-distal direction and (2) yielded a response (mean impulse rate) to the final $\mathrm{P} \rightarrow \mathrm{D}$ scan that was within $15 \%$ of the original $\mathrm{P} \rightarrow \mathrm{D}$ scan. The distribution of evoked 
Figure 11. Effect of scanning direction on mean firing rate. Twenty-six neurons studied with eight scanning directions are shown. Left panel, Mean firing rate versus scanning direction. The direction yielding the largest mean firing rate ("best direction") is shown at the middle of the plot. Right panel, The abscissa is the overall mean firing rate (average firing rate over all scanning directions). The ordinate is the ratio of the firing rate in the best direction to the firing rate in the opposite direction. The dashed curve in the left panel illustrates one of the most selective responses (mean rate, 10 ips; directional index, 3.3).
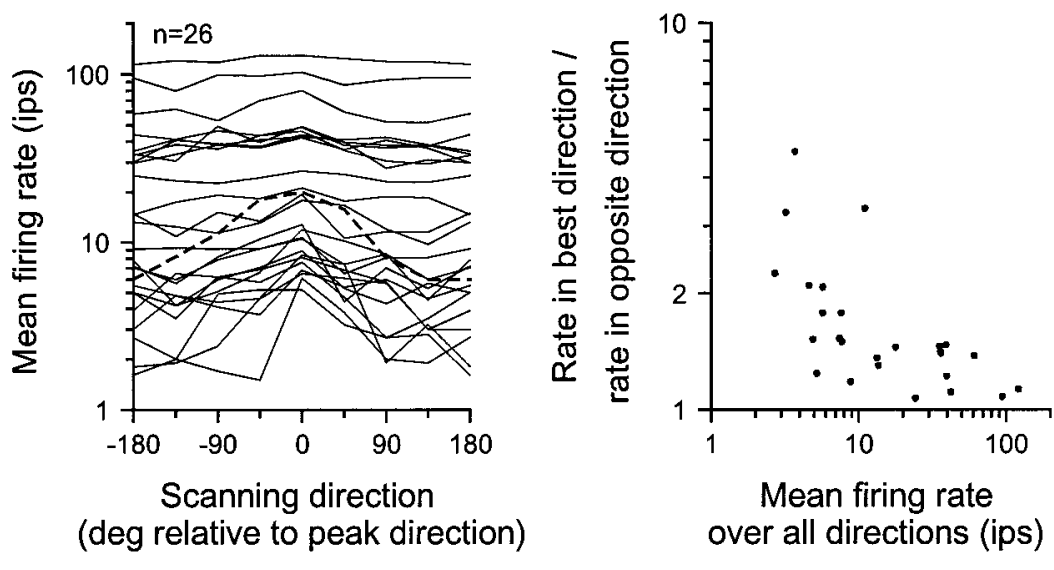

rates in this sample is broad, with mean firing rates varying by two orders of magnitude, as in the larger sample from area $3 \mathrm{~b}$ (DiCarlo and Johnson, 1999). The data in Figure 11 are qualitatively like the larger sample, which involved fewer than eight scan directions or did not include a repeated scan in the initial direction. A directional response metric was computed for each neuron as the firing rate in the "best" scanning direction divided by the firing rate in the opposite scanning direction (Fig. 11, right panel). A value of 1 indicates no directional sensitivity, and large values indicate strong directional sensitivity. Neurons with low evoked firing rates appeared to exhibit greater directional sensitivity, but that finding may reflect the fact that a small change in firing rates can produce a large change in a ratio measure when the rates are low. At higher rates (e.g., evoked mean rates of $\geq 10 \mathrm{ips}$ ), apparent directional sensitivity was less common, although a few neurons appeared to be selective; for example, among the 12 neurons with evoked rates $>10$ ips illustrated in Figure 11, one had a directional response ratio of 3.3, whereas the rest had ratios $<1.5$ (mean, 1.30). The curve for that neuron is plotted with a bold, dashed line in the left graph of Figure 11.

\section{Orientation sensitivity}

It is clear from the responses of neurons to random-dot patterns that many neurons are sensitive to dot clusters with certain orientations (e.g., Fig. 1 and comparable rasters in DiCarlo et al., 1998; DiCarlo and Johnson, 1999). The question addressed here is whether the three-component model derived from the randomdot responses predicts the same neuron's response to bars scanned across the RF with different orientations. We scanned oriented bars over the RFs of 67 neurons in eight evenly spaced directions with the same drum stimulator used to scan the random-dot pattern (see Materials and Methods). An example of the response of one area $3 \mathrm{~b}$ neuron (also illustrated in Figs. 1, 3) to a scanned bar is shown in the Figure 12, top panel. This neuron responded most strongly to bars scanned from right to left and from distal-left to proximal-right and responded least strongly to the orthogonal orientations. Ellipses were fitted to the polar data (see Fig. 12, Materials and Methods) to obtain a quantitative estimate of orientation sensitivity and the best orientation, if any. The ratio of the major to minor axes (aspect ratio) of the fitted ellipse provides an index of the orientation sensitivity; for example, the neuron illustrated in Figure 12 had an orientation sensitivity index of 3.1, which indicates that it responded 3.1 times more strongly to a bar aligned in its preferred orientation than to a bar aligned in the orthogonal orientation. The observed orientation sensitivities for the entire sample are illustrated in Figure
12 , bottom left histogram. Twenty-six of the 67 neurons tested had orientation sensitivities $>1.5$. The orientation of the minor axis of the fitted ellipse corresponds to the orientation of the bar producing the strongest response. The preferred orientations of 49 neurons with orientation sensitivities $>1.2$ is shown Figure 12, bottom right histogram. The distribution of orientations is reasonably uniform except for a deficit near $90^{\circ}$ (orientation along the finger axis, $p=0.02$, Kolmogorov-Smirnov).

How well does the three-component RF model derived in the first part of this paper predict the observed neural responses to scanned, oriented bars? We used each of the 62 three-component models to predict each neuron's response to a bar scanned in 16 evenly spaced directions and summarized these predicted responses with an ellipse in exactly the same way as the observed responses. The distributions of predicted orientation sensitivities and preferred orientations (data not shown) were similar to the distributions shown in Figure 12, bottom, which indicates that scanned, oriented bars do not produce orientation sensitivity beyond that predicted by linear RFs. The predicted orientation sensitivity was most strongly dependent on the strength of the fixed inhibition in comparison with the excitatory mass; the correlation between predicted orientation sensitivity and the ratio between fixed inhibitory and excitatory mass was $0.46(p<$ 0.001 ). Of these 62 neurons, 24 were also studied with scanned, oriented bars. The left middle scatter plot in Figure 12 shows that the predicted orientation sensitivity was within $50 \%$ of the observed orientation sensitivity for $96 \%$ (23 of 24) of these neurons (Pearson's correlation coefficient $=0.714 ; p<0.01$ ). Nineteen of these 24 neurons had observed orientation sensitivities $>1.2$, and the right middle scatter plot in Figure 12 shows that, for these neurons, the preferred orientation predicted by the threecomponent RF model was strongly related to the observed preferred orientation. If the observed and predicted orientations were unrelated, the differences between them would be uniformly distributed between -90 and $+90^{\circ}$; in fact, $79 \%$ (15 of 19) of these neurons had predicted preferred orientations within $45^{\circ}$ of the observed preferred orientation ( $p=0.002)$.

In summary, area $3 b$ neurons exhibit a range of orientation sensitivities to scanned bars, and that range is consistent with the range of sensitivities predicted by the three-component RF models determined with scanned random dots. For most neurons, the three-component RF model provides a good description of the neuron's sensitivity to orientation and its preferred orientation. The ability of each neuron's three-component RF model to predict both the degree of orientation sensitivity and the preferred 

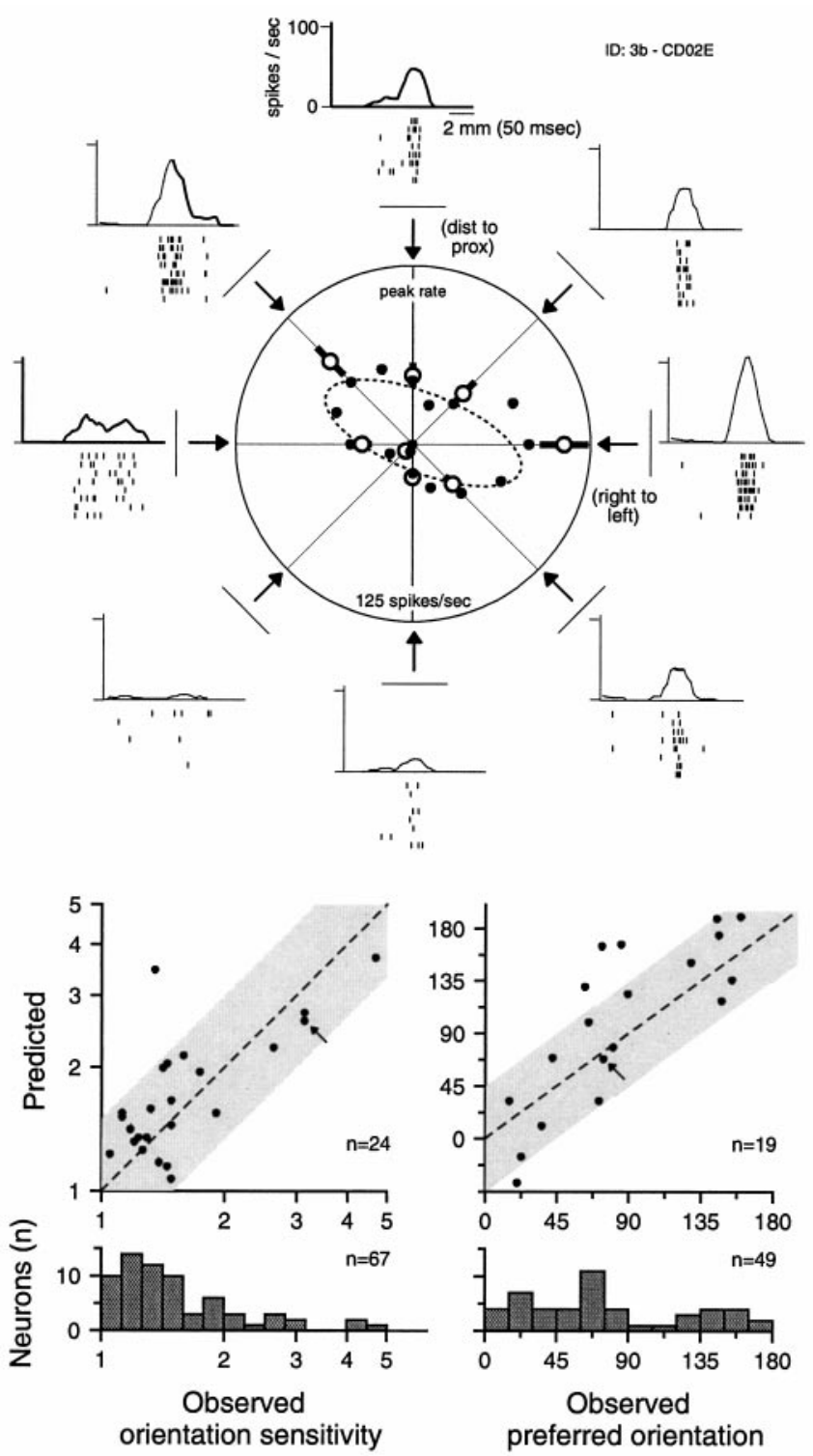

Figure 12. Orientation sensitivity and its prediction by the threecomponent model. The top panel illustrates the responses of one of the more orientation-selective neurons that was also studied with the randomdot patterns. Each raster plot shows spikes (tick marks) produced in response to eight repeated scans of a single, raised bar scanned in a particular direction across the neuron's RF. The histogram above each raster shows the spike data binned across trials and filtered (see Materials and Methods). The peak value of each histogram was taken as the neuron's response in that scanning direction and these values are plotted as open circles along the radial lines in the middle polar plot (error bars are SDs computed by bootstrap; Efron and Tibshirani, 1993). The 16 filled circles show the responses predicted by the three-component model for this neuron (same neuron as in Fig. 3) in 16 directions. The dashed line shows the ellipse that best fits the 16 predicted response values (leastsquared radial error). The middle left scatter plot shows the observed orientation sensitivity (ellipse aspect ratio) on the abscissa and the predicted orientation sensitivity on the ordinate for 24 neurons whose threecomponent RF models and orientation sensitivities were determined. The middle right scatter plot shows the observed preferred orientation (ellipse angle) on the abscissa and the predicted preferred orientation on the ordinate for 19 of these 24 neurons whose observed orientation sensitivities were $>1.2$. The small arrow in each scatter plot indicates the datum from the neuron illustrated in the top panel. The bottom two panels show the distributions of observed orientation sensitivities and observed preferred orientations (for neurons with orientation sensitivities $>1.2$ ). See Materials and Methods and Results for details.

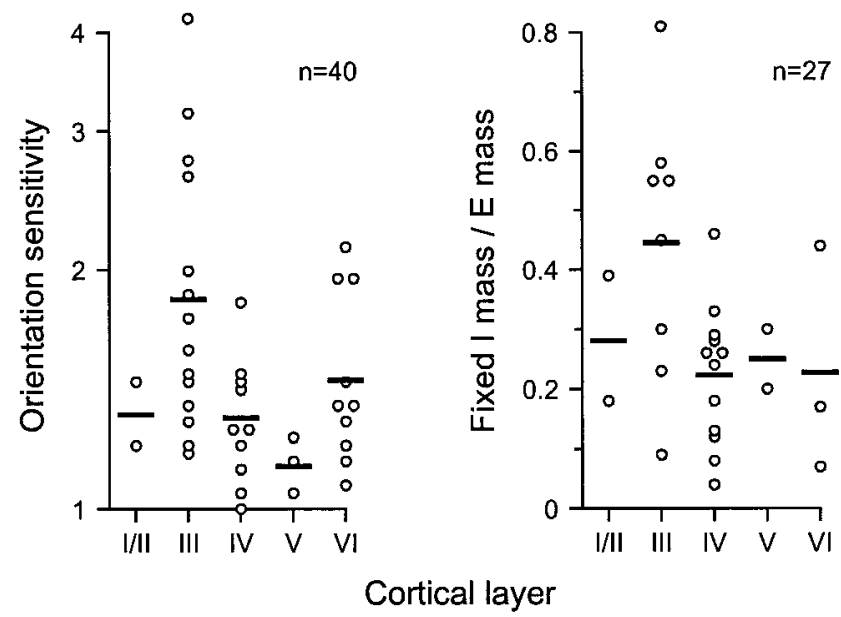

Figure 13. Relationship of orientation sensitivity and RF mass ratio to cortical layer. The abscissa of both plots is the cortical layer in which each neuron was recorded (see Materials and Methods). The ordinate of the left plot is the observed orientation sensitivity (fitted ellipse aspect ratio; see Results). Forty area $3 b$ neurons whose cortical layer and orientation sensitivity were both determined are shown. The ordinate of the right plot is the ratio of the mass of the fixed inhibitory RF component and the mass of the excitatory RF component. Twenty-seven area $3 b$ neurons whose cortical layer and three-component RF models were both determined are shown. The thick bars indicate the mean value in each cortical layer.

orientation suggests that it has indeed captured some of the salient neural response properties. This is especially striking considering that (1) the three-component models are "metamodels" in that they are condensed descriptions of RF estimates that are themselves incomplete (i.e., linear) descriptions of the actual neural responses (see DiCarlo et al., 1998, Fig. 13), and (2) the RF estimates (and thus the three-component models) were derived from the neural responses to a scanned, random-dot stimulus, which does not contain the bar stimuli used to test the orientation sensitivity.

\section{Relationship to cortical layer}

The results of this study and our previous studies (DiCarlo et al., 1998; DiCarlo and Johnson, 1999) suggest that the range of neural RFs found in area $3 \mathrm{~b}$ underlies a range of response selectivities for particular spatiotemporal tactile patterns (e.g., see DiCarlo et al., 1998, their Fig. 14). Given the extensive anatomical and physiological data suggesting that increasingly complex response properties might be elaborated between layer IV (granular layer) and supragranular layers (see Discussion), we hypothesized that the degree of neural response selectivity and the RF properties that underlie that selectivity might be related to each neuron's laminar position. Specifically, if some of the response selectivity is due to intracortical processing, then neurons with the least selective responses and "simplest" RFs should be found in the area $3 b$ layer that receives thalamocortical projections (i.e., the granular layer; Jones and Burton, 1976), and neurons with the most selective responses and most complex RFs should be found in the area $3 b$ layers that project to higher cortical areas (i.e., the supragranular layers). One example of the range of spatial selectivities observed in area $3 \mathrm{~b}$ is the range of orientation sensitivities described in the previous section. Indeed, we found that the degree of orientation sensitivity of each area $3 \mathrm{~b}$ neuron is strongly related to its laminar position. Figure 13 shows the orientation sensitivity (ellipse aspect ratio) of 40 neurons (of 67 studied for orientation sensitivity) whose laminar recording loca- 
tions were determined (see Materials and Methods). The plot shows that the neurons with the strongest orientation sensitivities tend to occur in layer III and perhaps layer VI. The mean orientation sensitivity in the supragranular layers (I-III) was significantly greater than in both the granular layer (means, 1.91 and 1.32 respectively; $t$ test, $t=2.78 ; p=0.011$ ) and the infragranular layers (mean, $1.41 ; t=2.18 ; p=0.039$ ).

Because the orientation sensitivity of area $3 \mathrm{~b}$ neurons was reasonably well predicted by their three-component RF descriptions (Fig. 12), we wondered what aspects of each neuron's three-component RF might also be related to cortical layer. This question was examined in 27 of the 62 neurons described in the first part of this paper that were localized accurately to a layer within area 3b. Of these 27 neurons, 5 were infragranular, 12 were in layer IV, and the rest were supragranular. The mean granular and infragranular values for all the RF components were similar, and no clear relationships were evident. Between layer IV and the supragranular layers the geometric mean area and mass changed marginally (by +7 and $-17 \%$, respectively), as did the mean lagged inhibitory area and mass (by -5 and $-20 \%$, respectively). In contrast, the fixed inhibitory area and mass both increased substantially (by 69 and 56\%, respectively). As a result, the ratio of the fixed inhibitory area and mass to excitatory area and mass increased between layer IV and the supragranular layers by 62 and $87 \%$, respectively. Because of the correction required for multiple comparisons (Bonferroni correction), none of the findings reached statistical significance. If the change in the ratio of fixed inhibitory to excitatory mass were considered on its own, it would have been significant ( $p=0.046 ; t=2.2$ ). Because strong, spatially fixed regions of RF inhibition can have powerful effects on a neuron's sensitivity to spatial form, it is not surprising that the RF parameter most strongly related to each neuron's laminar position is the intensity (mass) of its spatially fixed inhibitory component in comparison with its excitatory component. This relationship is shown in Figure 13, right scatter plot.

\section{DISCUSSION}

A result of the present study and two previous studies with scanned, random-dot patterns (DiCarlo et al., 1998; DiCarlo and Johnson, 1999) is that the linear part of the responses of area $3 b$ neurons is described by RFs with three components: (1) a single, central excitatory region of short duration, (2) one or more inhibitory regions that are adjacent to and nearly synchronous with the excitation, and (3) an inhibitory region that overlaps the excitation partially or totally and is delayed with respect to the first two components. This description accounts well for the findings of all three studies. The principal finding of the first study was the wide range of RFs in area 3b (DiCarlo et al., 1998). Figures 3-7 in this paper show how such a wide range of RFs emerge from the three-component model. The first study, which used proximal-todistal scanning, also showed that in most cases (87\%) the center of inhibitory mass was displaced distally from the center of excitation. This suggested either that there was a bias in the location of inhibition or that inhibition lagged behind the excitation in the scanning direction. The present study shows that both are true: a temporal lag accounts for most of the bias in the first study, but it is also true that in 42 of the 62 RFs studied here the center of fixed inhibition was displaced distally from the center of excitation. The principal finding of the second study was that RF spatial structure is virtually invariant with changes in scanning velocity (DiCarlo and Johnson, 1999). Analyses accompanying the second study showed that this invariance requires either brevity and synchrony in all the excitatory and inhibitory components or overlap between the excitation and the lagged inhibition (see DiCarlo and Johnson, 1999, their Fig. 11). The present study supports both hypotheses: some of the inhibition is brief and synchronous with the excitation; the remaining lagged inhibition overlaps the excitation in exactly the manner that is required for velocity invariance. The principal empirical finding of the present study is that area $3 \mathrm{~b}$ RFs are strongly affected by scanning direction. Goodness-of-fit analyses showed that the threecomponent model provides an accurate fit to these RFs (see Fig. 8). The generality of the model was shown (as in DiCarlo et al., 1998) by comparing the responses to stimuli not used to estimate the RFs or the model (oriented bars in this case) with predicted responses.

\section{Excitatory component}

The removal of intracortical inhibition by the administration of GABA antagonists increases the excitatory area and intensity of area $3 \mathrm{~b}$ responses as the three-component model suggests (Dykes et al., 1984; Alloway and Burton, 1991), but the expansion (200$300 \%$ ) is much larger than would be predicted from the model. One explanation for this discrepancy is that the excitatory and inhibitory drives are more extensive than we have shown but are perfectly balanced outside the regions we have identified. The more likely explanation is that a GABA antagonist injected into the extracellular space unmasks excitatory drive that does not normally impinge on the neuron being studied. The increased latency of excitation after GABA antagonism is consistent with this explanation (Alloway and Burton, 1991).

Whatever is the extent of the excitatory drive, the invariance of the spatial structure of excitatory subfields with changes in both direction and velocity suggests that the excitatory drive is brief. Analyses of the effects of velocity changes on excitatory RF area in the second study (DiCarlo and Johnson, 1999) suggested that excitation persists for $\leq 10 \mathrm{msec}(\mathrm{SD})$.

\section{Fixed inhibitory component}

The fixed inhibitory component was usually a single region displaced to one side of the excitation, but occasionally more than one such region was observed. Because its location and shape were unaffected by changes in both scanning velocity and direction, we infer that the fixed inhibition is brief and synchronous with the excitation. It must also be of central origin, because neither SA1 nor RA afferents exhibit suppression at a fixed skin location independent of scanning direction (DiCarlo and Johnson, unpublished observations). Evidence that at least some of the fixed inhibitory effects are due to intracortical mechanisms comes from the laminar analyses, which showed that the fixed inhibitory component was strongest (in comparison with the excitatory component) in the supragranular layers (Fig. 13).

\section{Lagged inhibitory component}

The lagged inhibitory component may be partly the result of SA1 primary afferent response properties. SA1 afferents exhibit lagged suppression (one dot suppresses the response to a second dot trailing behind) but its mass (43\% of the excitatory mass on average; DiCarlo and Johnson, 1999) is only approximately half as great as the lagged inhibitory mass in area $3 \mathrm{~b}$ (mean, 79\%; range, $14-157 \%$ ). RA afferents exhibit almost no lagged suppression (mean, 6\%) and therefore cannot account, even partially, for the lagged inhibition reported here.

The portion of the lagged inhibition that is due to central mechanisms is almost certainly the infield inhibition studied by 
Laskin and Spencer (1979) and Gardner and Costanzo (1980a). They used conditioning and test stimuli to reveal inhibition that overlaps excitation, which is maximal at $10-20 \mathrm{msec}$ after the peak of excitation and persists for $\geq 50 \mathrm{msec}$. Our estimates of the delay between the excitation and the temporal center of lagged inhibition (mean, $29 \mathrm{msec}$ ) are consistent with those previous observations. Temporally lagged inhibition that overlaps excitation has been shown in other ways. Intracellular studies have revealed temporally delayed, spatially overlapped hyperpolarization (i.e., true inhibition, not response suppression) in neurons in the cuneate nucleus (Andersen et al., 1964a; Jänig et al., 1977), thalamus (Andersen et al., 1964b; Iwamura and Inubushi, 1974; Jänig et al., 1979), and SI cortex (Andersson, 1965; Whitehorn and Towe, 1968; Innocenti and Manzoni, 1972). Extensive overlap between excitation and inhibition is also suggested by the administration of GABA antagonists (Dykes et al., 1984; Alloway and Burton, 1991).

\section{Functional implications}

The fixed excitatory and inhibitory components of each neuron function as a spatial filter, conferring selectivity for particular spatial features or patterns regardless of scanning direction and velocity. The lagged inhibitory component confers selectivity for stimulus gradients in the scanning direction, regardless of that direction. To the extent that its lag center is displaced from the center of excitation, it also functions, at least theoretically, as a basis for directional sensitivity. These are the functional implications for linear mechanisms; they must be tempered by the knowledge that a linear description is incomplete. The test is their explanatory power.

Directional sensitivity is one such test. When the stimulus is scanned in a direction defined by a vector from the center of excitation to the lag center (Fig. 2), the lagged inhibition is displaced away from the excitation and uncovers it maximally; this should maximize the firing rate. In the opposite direction the lagged inhibition swings over the excitation and masks it maximally; this should minimize the firing rate (cf., Barlow and Levick, 1965; Gardner and Costanzo, 1980b; Warren et al., 1986). The lag offsets were generally small in our study (mean, $0.35 \mathrm{~mm}$ ), which minimizes this effect, but three neurons had offsets close to $1 \mathrm{~mm}$ (Fig. 10). These three neurons also had higher response rates for the scanning direction predicted by the lag offset. The generally small lag offsets (Fig. 10) and mild directional selectivities observed in the present study (Fig. 11) are consistent with previous reports of mild to moderate directional sensitivity (Costanzo and Gardner, 1980; Warren et al., 1986; Ruiz et al., 1995).

Selectivity for spatial form is a more important test of the three-component description. Raster plots of the responses of area $3 b$ neuron to scanned, complex patterns such as raised letters show that many neurons are selective for the features of complex stimuli (Phillips et al., 1988; Bankman et al., 1990; DiCarlo et al., 1998) and that these responses are explained in part by linear RFs (Johnson et al., 1995; DiCarlo et al., 1998). Also, neurons in area 3b are moderately orientation-selective (Pubols and LeRoy, 1977; Hyvarinen and Poranen, 1978; Warren et al., 1986). We confirmed this observation and showed that this orientation sensitivity is predicted by the three-component RF model (Fig. 12). The RF component most strongly associated with orientation sensitivity was the fixed inhibition.

We also found that orientation sensitivity and strong, spatially offset RF inhibition (in comparison with excitation) were more common in the infragranular and supragranular layers than in layer IV (Fig. 13). Twelve of 40 neurons whose laminar locations were identified had orientation selectivities $>1.5$, and all but one of those were in the infragranular or supragranular layers. Anatomical literature demonstrates strong excitatory pathways between layers (e.g., granular to supragranular projections) within area 3b (Schwark and Jones, 1989) and shows that projections to higher cortical areas (e.g., SII) arise mainly from the supragranular layers of area 3b (Jones, 1984). Other physiological studies have revealed larger RF sizes (Sur et al., 1985) and more complex spatial response properties (DiCarlo et al., 1996) in supragranular (vs granular) layers. All of this suggests that selectivity for spatial forms (e.g., oriented edges) increases within area $3 b$ and that this increasingly nonisomorphic representation of tactile spatial form is relayed to higher cortical areas.

\section{REFERENCES}

Alloway KD, Burton H (1991) Differential effects of GABA and bicuculline on rapidly- and slowly-adapting neurons in primary somatosensory cortex of primates. Exp Brain Res 85:598-610.

Andersen P, Eccles JC, Oshima T, Schmidt RF (1964a) Mechanisms of synaptic transmission in the cuneate nucleus. J Neurophysiol 27:1096-1116.

Andersen P, Eccles JC, Sears TA (1964b) The ventrobasal complex of the thalamus: types of cells, their responses and their functional organization. J Physiol (Lond) 174:370-399.

Andersson SA (1965) Intracellular postsynaptic potentials in the somatosensory cortex of the cat. Nature 205:297-298.

Bankman IN, Johnson KO, Hsiao SS (1990) Neural image transformation in the somatosensory system of the monkey: comparison of neurophysiological observations with responses in a neural network model. Cold Spring Harb Symp Quant Biol 55:611-620.

Barlow HB, Levick WR (1965) The mechanism of directionally selective units in rabbit's retina. J Physiol (Lond) 178:477-504.

Costanzo RM, Gardner EP (1980) A quantitative analysis of responses of direction-sensitive neurons in somatosensory cortex of awake monkeys. J Neurophysiol 43:1319-1341.

DiCarlo JJ, Johnson KO (1999) Velocity invariance of receptive field structure in somatosensory cortical area $3 \mathrm{~b}$ of the alert monkey. J Neurosci 19:401-419.

DiCarlo JJ, Lane JW, Hsiao SS, Johnson KO (1996) Marking microelectrode penetrations with fluorescent dyes. J Neurosci Methods 64:75-81.

DiCarlo JJ, Johnson KO, Hsiao SS (1998) Structure of receptive fields in area $3 \mathrm{~b}$ of primary somatosensory cortex in the alert monkey. J Neurosci 18:2626-2645.

Dykes RW, Landry P, Metherate RS, Hicks TP, Metherate R (1984) Functional role of GABA in cat primary somatosensory cortex: shaping receptive fields of cortical neurons. J Neurophysiol 52:1066-1093.

Efron B, Tibshirani RJ (1993) An introduction to the bootstrap. London: Chapman and Hall.

Gardner EP, Costanzo RM (1980a) Temporal integration of multiplepoint stimuli in primary somatosensory cortical receptive fields of alert monkeys. J Neurophysiol 43:444-468.

Gardner EP, Costanzo RM (1980b) Neuronal mechanisms underlying direction sensitivity of somatosensory cortical neurons in awake monkeys. J Neurophysiol 43:1342-1354.

Hyvarinen J, Poranen A (1978) Movement-sensitive and direction and orientation-selective cutaneous receptive fields in the hand area of the post-central gyrus in monkeys. J Physiol (Lond) 283:523-537.

Innocenti GM, Manzoni T (1972) Response patterns of somatosensory cortical neurones to peripheral stimuli. An intracellular study. Arch Ital Biol 110:322-347.

Iwamura Y, Inubushi S (1974) Regional diversity in excitatory and inhibitory receptive-field organization of cat thalamic ventrobasal neurons. J Neurophysiol 37:910-919.

Jänig W, Schoultz T, Spencer WA (1977) Temporal and spatial parameters of excitation and afferent inhibition in cuneothalamic relay neurons. J Neurophysiol 40:822-835.

Jänig W, Spencer WA, Younkin SG (1979) Spatial and temporal features of afferent inhibition of thalamocortical relay cells. J Neurophysiol 42:1450-1460.

Johnson KO, Phillips JR (1988) A rotating drum stimulator for scanning 
embossed patterns and textures across the skin. J Neurosci Methods 22:221-231.

Johnson KO, Hsiao SS, Twombly IA (1995) Neural mechanisms of tactile form recognition. In: The cognitive neurosciences (Gazzaniga MS, ed), pp 235-268. Cambridge, MA: MIT.

Jones EG (1984) Laminar distribution of cortical efferent cells. In: Cerebral cortex. Vol 1, Cellular components of the cerebral cortex (Peters A, Jones EG, eds), pp 521-554. New York: Plenum.

Jones EG, Burton H (1976) Areal differences in the laminar distribution of thalamic afferents in cortical fields of the insular, parietal and temporal regions of primates. J Comp Neurol 168:197-248.

Laskin SE, Spencer WA (1979) Cutaneous masking. II. Geometry of excitatory and inhibitory receptive fields of single units in somatosensory cortex of the cat. J Neurophysiol 42:1061-1082.

Mardia KV (1972) Statistics of directional data. London: Academic.

Mountcastle VB, Reitboeck HJ, Poggio GF, Steinmetz MA (1991) Adaptation of the Reitboeck method of multiple microelectrode recording to the neocortex of the waking monkey. J Neurosci Methods 36:77-84.

Phillips JR, Johnson KO, Hsiao SS (1988) Spatial pattern representation and transformation in monkey somatosensory cortex. Proc Natl Acad Sci USA 85:1317-1321.

Powell TPS, Mountcastle VB (1959) The cytoarchitecture of the post- central gyrus of the monkey Macaca mulatta. Bull Johns Hopkins Hosp 105:108-131.

Press WH, Teukolsky SA, Vetterling WT, Flannery BP (1992) Numerical recipes in FORTRAN: the art of scientific computing. Cambridge, UK: Cambridge UP.

Pubols Jr BH, LeRoy RF (1977) Orientation detectors in the primary somatosensory neocortex of the raccoon. Brain Res 129:61-74.

Ruiz S, Crespo P, Romo R (1995) Representation of moving tactile stimuli in the somatic sensory cortex of awake monkeys. J Neurophysiol 73:525-537.

Schwark HD, Jones EG (1989) The distribution of intrinsic cortical axons in area $3 \mathrm{~b}$ of cat primary somatosensory cortex. Exp Brain Res 78:501-513.

Sur M, Garraghty PE, Bruce CJ (1985) Somatosensory cortex in macaque monkeys: laminar differences in receptive field size in areas $3 b$ and 1. Brain Res 342:391-395.

Warren S, Hämäläinen HA, Gardner EP (1986) Objective classification of motion- and direction-sensitive neurons in primary somatosensory cortex of awake monkeys. J Neurophysiol 56:598-622.

Whitehorn D, Towe AL (1968) Postsynaptic potential patterns evoked upon cells in sensorimotor cortex of cat by stimulation at the periphery. Exp Neurol 22:222-242. 